\title{
High-energy Emission from Pulsar Outer Magnetospheres
}

\author{
Kouichi Hirotani ${ }^{1}$ \\ Code 661.0, Laboratory for High Energy Astrophysics, \\ NASA/Goddard Space Flight Center, \\ Greenbelt, MD 20771; \\ Email: hirotani@mpi-hd.mpg.de
}

To appear in the inaugural issue of Progress in Astrophysics Researches

\begin{abstract}
We investigate a stationary pair production cascade in the outer magnetosphere of an isolated, spinning neutron star. The charge depletion due to global flows of charged particles, causes a large electric field along the magnetic field lines. Migratory electrons and/or positrons are accelerated by this field to radiate gamma-rays via curvature and inverse-Compton processes. Some of such gamma-rays collide with the X-rays to materialize as pairs in the gap. The replenished charges partially screen the electric field, which is self-consistently solved together with the energy distribution of particles and gamma-rays at each point along the field lines. By solving the set of Maxwell and Boltzmann equations, we demonstrate that an external injection of charged particles at nearly Goldreich-Julian rate does not quench the gap but shifts its position and that the particle energy distribution cannot be described by a power-law. The injected particles are accelerated in the gap and escape from it with large Lorentz factors. We show that such escaping particles migrating outside of the gap contribute significantly to the gamma-ray luminosity for young pulsars and that the soft gamma-ray spectrum between $100 \mathrm{MeV}$ and $3 \mathrm{GeV}$ observed for the Vela pulsar can be explained by this component. We also discuss that the luminosity of the gamma-rays emitted by the escaping particles is naturally proportional to the square root of the spin-down luminosity.
\end{abstract}

Subject headings: gamma-rays: observations - gamma-rays: theory - magnetic fields methods: numerical - pulsars: individual (Geminga pulsar, PSR B1055-52, PSR B170644, Vela pulsar)

\footnotetext{
${ }^{1}$ Present address: Max-Planck-Institut für Kernphysik, Postfach 103980, D-69029 Heidelberg, Germany
} 


\section{Introduction}

Recent years have seen a renewal of interest in the theory of particle acceleration in pulsar magnetospheres, after the launch of the Compton Gammaray Observatory (CGRO). The Energetic Gamma Ray Experiment Telescope (EGRET) on board the CGRO has detected pulsed signals from at least seven rotation-powered pulsars (for the Crab pulsar, Nolan et al. 1993, Fierro et al. 1998; for the Vela pulsar, Kanbach et al. 1994, Fierro et al. 1998; for PSR B1706-44, Thompson et al. 1996; for PSR B1951, Ramanamurthy et al. 1995; for PSR B1046-58, Kaspi at al. 2000; for Geminga, Mayer-Hasselwander et al. 1994, Fierro et al. 1998; for PSR B1055-52, Thompson et al. 1999). Since interpreting $\gamma$-rays should be less ambiguous compared with reprocessed, non-thermal X-rays, the $\gamma$-ray pulsations observed from these objects are particularly important as a direct signature of basic non-thermal processes in pulsar magnetospheres, and potentially should help to discriminate among different emission models.

Attempts to model the pulsed $\gamma$-ray emissions have concentrated on two scenarios (fig. 1): Polar cap models with emission altitudes of $\sim 10^{4} \mathrm{~cm}$ to several neutron star radii over a pulsar polar cap surface (Harding, Tademaru, \& Esposito 1978; Daugherty \& Harding 1982, 1996; Dermer \& Sturner 1994; Sturner, Dermer, \& Michel 1995; also see Scharlemann, Arons, \& Fawley 1978 for the slot gap model), and outer gap models with acceleration occurring in the open field zone located near the light cylinder (Cheng, Ho, \& Ruderman 1986a,b, hereafter CHRa,b; Chiang \& Romani 1992, 1994; Romani and Yadigaroglu 1995; Romani 1996). Both models predict that electrons and positrons are accelerated in a charge depletion region, a potential gap, by the electric field along the magnetic field lines to radiate high-energy $\gamma$-rays via the curvature process. However, there is an important difference between these two models: An polargap accelerator releases very little angular momenta, while an outer-gap one could radiate them efficiently. In addition, three-dimensional outer-gap models commonly explain double-peak light curves with strong bridges observed for the $\gamma$-ray pulsars. The purpose of the present paper is, therefore, to explore further into the analysis of the outer-gap models.

If a magnetized neutron star is rotating with angular velocity $\Omega$, a static observer measures the rotation- ally induced charge density (Goldreich \& Julian 1969; Mestel 1971)

$$
\rho_{\mathrm{GJ}} \equiv-\frac{1}{4 \pi c} \nabla \cdot[(\boldsymbol{\Omega} \times \boldsymbol{r}) \times \boldsymbol{B}],
$$

where $\boldsymbol{\Omega}$ satisfies $|\boldsymbol{\Omega}|=\boldsymbol{\Omega}$ and directs the rotation axis (fig. 1), $\boldsymbol{B}$ is the magnetic field at potision $\boldsymbol{r}$ from the stellar center, $c$ the speed of light. Expanding the right-hand side with respect to $|\boldsymbol{\Omega} \times \boldsymbol{r}| / c$, we obtain

$$
\rho_{\mathrm{GJ}}=-\frac{\Omega B_{\zeta}}{2 \pi c}\left[1+O(\boldsymbol{\Omega} \times \boldsymbol{r} / c)^{2}\right],
$$

where $B_{\zeta} \equiv \boldsymbol{B} \cdot \boldsymbol{\Omega} / \Omega$. For a Newtonian dipole magnetic field, the null surface, where $B_{\zeta}$ and hence $\rho_{\mathrm{GJ}}$ vanishes, is located on a constant colatitude in the outer magnetosphere on the poloidal plane (heavy dashed line in fig. 1).

If the real charge density, $\rho_{\mathrm{e}}$, deviates from $\rho_{\mathrm{GJ}}$ in any region, an electric field is exerted along $\boldsymbol{B}$. If the potential drop is sufficient, migratory electrons and/or positrons will be accelerated to radiate $\gamma$ rays via curvature and/or inverse-Compton (IC) processes. Some of such $\gamma$-rays collide with the soft photons illuminating the outer part of the magnetosphere and materialize as pairs in the gap. The replenished charges partially screen the original acceleration field, $E_{\|} \equiv \boldsymbol{E} \cdot \boldsymbol{B}_{\mathrm{p}} / B_{\mathrm{p}}$, where $\boldsymbol{B}_{\mathrm{p}}$ is the magnetic field projected on the poloidal plane, and $B_{\mathrm{p}} \equiv\left|\boldsymbol{B}_{\mathrm{p}}\right|$. If the created particles pile up at the boundaries of the potential gap, they will quench the gap eventually. Nevertheless, if the created particles continue to migrate outside of the gap as a part of the global flows of charged particles, a steady charge-deficient region could be maintained. This is the basic idea of a particle acceleration zone in a pulsar magnetosphere.

In the CHR picture, the mainstream of the outergap model, the gap is hypothesized to be geometrically thin in the transfield direction on the poloidal plane in the sense $D_{\perp} \ll W$, where $D_{\perp}$ represents the typical transfield thickness of the gap, while $W$ does the width along the magnetic field lines. In this limit, the acceleration electric field is partially screened by the zero-potential walls separated with a small distance $D_{\perp}$; as a result, the gap, which is assumed to be vacuum, extends from the null surface to (the vicinity of) the light cylinder (fig. 1).

If $B_{\zeta}>0$ holds in the starward side of the null surface, a positive acceleration field arises in the gap. The light cylinder is defined as the surface where the azimuthal velocity of a plasma would coincide with $c$ 
if it corotated with the magnetosphere. Its distance, $\varpi_{\mathrm{LC}} \equiv c / \Omega$, from the rotational axis is called the 'light cylinder radius'. Particles are not allowed to migrate inwards beyond this surface because of the causality in special relativity.

It should be noted that the null surface (where $B_{\zeta}$ vanishes) is not a special place for the gap electrodynamics in the sense that the plasmas are not completely charge-separated in general and that the particles freely pass through this surface inwards and outwards. Therefore, the gap inner boundary is located near to the null surface, not because a particle injection is impossible across this surface (as previously discussed), but because the gap is vacuum and transversely thin.

Then what happens in the CHR picture if the gap becomes no longer vacuum? To consider this problem rigorously, we have to examine the Poisson equation for the electrostatic potential. In fact, as will be explicitly demonstrated in the next section, the original vacuum solution obtained in the pioneering work by CHR cannot be applied to a non-vacuum CHR picture. We are, therefore, motivated by the need to solve self-consistently the Poisson equation together with the Boltzmann equations for particles and $\gamma$-rays. Although the ultimate goal is to solve three-dimensional issues, a good place to start is to examine one-dimensional problems. In this context, Hirotani and Shibata (1999a, b, c; hereafter Papers I, II, III) and Hirotani (2000b, Paper VI) first solved the Boltzmann equations together with the Maxwell equations one-dimensionally along the field lines, extending the idea originally developed for black-hole magnetospheres by Beskin et al. (1992). Subsequently, Hirotani (2000a, Paper IV; 2001, Paper V) considered a 'gap closure condition' (eq. [29]) to constrain the gap width and estimated the $\gamma$-ray fluxes for individual pulsars.

There is one important finding in this second picture: The gap position shifts if there is a particle injection across either of the boundaries (Hirotani \& Shibata 2001, 2002a,b; hereafter Papers VII, VIII, IX). For example, when the injection rate across the outer (or inner) boundary becomes comparable to the typical Goldreich-Julian value, the gap is located close to the neutron star surface (or to the light cylinder). In other words, an outer gap is not quenched even when the injection rate of a completely chargeseparated plasma across the boundaries approaches the typical Goldreich-Julian value. Thus, an outer gap can coexist with a polar-cap accelerator; this forms a striking contrast to the first, CHR picture. It is also found in the second picture that an outer gap is quenched if the created particle density within the gap exceeds some fractions of the Goldreich-Julian value. That is, the discharge of created pairs is essential to screen the acceleration field.

More recently, Hirotani, Harding, and Shibata (2003, Paper X) demonstrated that the particle energy distribution cannot be represented either by a power law or by the mono-energetic approximation, by solving explicitly the energy dependence of particle distribution functions, together with $E_{\|}$and the $\gamma$-ray distribution functions. They further showed that a soft power-law spectrum is generally formed in $100 \mathrm{MeV}-3 \mathrm{GeV}$ energies as a result of the superposition of the curvature spectra emitted by particles migrating at different positions.

In the present article, we sum up the main points that have been made in the second picture (from $\mathrm{Pa}$ pers I to X), which assumes that the gap is geometrically thick in the transfield direction in the sense $D_{\perp}>0.3 W$.

In the next section, we demonstrate that the charge distribution in the non-vacuum CHR model does not satisfy the Maxwell equation. We then analytically constrain the gap position in $\S 3$, and explicitly formulate the basic equations in $\S 4$ for quantitative analysis. We apply the theory to individual pulsars in $\S 5$. In the final section, we discuss the stability of the gap, evolution of the $\gamma$-ray luminosity vs. spin-down one, and the unification of the CHR and the second pictures in outer-gap models, as well as the unification of the outer-gap and the polar-cap models.

\section{Difficulties in Previous Outer-gap Models}

To elucidate the electrodynamic difficulties in previous outer-gap models, we have to examine the Poisson equation for the electrostatic potential. In the inertial frame, the Poisson equation becomes

$$
\nabla \cdot\left[-\frac{1}{c}(\boldsymbol{\Omega} \times \boldsymbol{r}) \times \boldsymbol{B}-\nabla \Psi\right]=4 \pi \rho_{\mathrm{e}}(\boldsymbol{r}),
$$

where the left-hand side is the divergence of the electric field. The real charge density, $\rho_{\mathrm{e}}$, is given by $\rho_{\mathrm{e}}=\rho_{+}+\rho_{-}$, where $\rho_{+}$and $\rho_{-}$represent the positronic and electronic charge densities, respectively. The non-corotational potential $\Psi$ is related to the usual scalar and vector potential $\left(A_{0}, \boldsymbol{A}\right)$ as 
$\Psi=A_{0}-(\Omega \varpi / c) \boldsymbol{e}_{\phi} \cdot \boldsymbol{A}$, where $\varpi$ designates the distance from the rotation axis, $\boldsymbol{e}_{\phi}$ the azimuthal unit vector. Noting that $\boldsymbol{E}_{\perp} \equiv-(\boldsymbol{\Omega} \times \boldsymbol{r}) \times \boldsymbol{B} / c$ represents the electric field perpendicular to the magnetic field, we find that the term $-\nabla^{2} \Psi$ is important for particle acceleration in equation (3).

Since the azimuthal dimension is supposed to be large compared with $D_{\perp}$ in conventional outer-gap models, equation (3) is reduced to the following twodimensional form on the poloidal plane

$$
-\frac{\partial^{2} \Psi}{\partial s^{2}}-\frac{\partial^{2} \Psi}{\partial z^{2}}=4 \pi\left[\rho_{\mathrm{e}}(s, z)-\rho_{\mathrm{GJ}}(s, z)\right],
$$

where equation (1) is used; $s$ and $z$ refer to the coordinates parallel and perpendicular, respectively, to the poloidal magnetic field. The star surface corresponds to $s=0 ; s$ increases outwardly along the field lines. The last-open field line corresponds to $z=0$; $z$ increases towards the magnetic axis (in the same hemisphere). A dipole magnetic field has a singlesigned curvature near the last open field line (i.e., at $z \ll \varpi_{\mathrm{LC}}$ ) except close to the light cylinder. Thus, $\gamma$-rays propagate into the higher altitudes (i.e., large $z$ regions); as a result, in the CHR picture, the particle number density $\left(\rho_{+}-\rho_{-}\right) / e$ grows exponentially in the $z$ direction, where $e$ designates the magnitude of the charge on an electron. Because of this exponential growth of the particle number density, it has been considered that most of the $\gamma$-rays are emitted from the higher altitudes.

To explain the observed $\gamma$-ray luminosities with a small $D_{\perp}$, one should assume that the created current density becomes the typical Goldreich-Julian value in the higher altitudes. That is, the conserved current density per magnetic flux tube should satisfy

$$
\frac{c \rho_{+}}{B}+\frac{-c \rho_{-}}{B} \sim \frac{\Omega}{2 \pi}
$$

in the order of magnitude, where $B \equiv|\boldsymbol{B}|$. However, such a copious pair production will screen the local acceleration field, $E_{\|}=-\partial \Psi / \partial s$, as the Poisson equation (4) indicates.

This screening effect is particularly important near to the inner boundary. Without loss of any generality, we can assume that $E_{\|}$is positive. In this case, because of the discharge, only electrons exist at the inner boundary. (We may notice here that external particle injections are not considered in the CHR picture.) Thus, we obtain $\rho_{\mathrm{e}} / B=\rho_{-} / B \sim-\Omega /(2 \pi c)$ in the order of magnitude. In the vicinity of the inner boundary, we can Fourier-analyze equation (4) in $z$ direction to find out that the $-\partial^{2} \Psi / \partial z^{2}$ term contributes only to reduce $\partial E_{\|} / \partial s=-\partial^{2} \Psi / \partial s^{2}$. Thus, a positive $-\rho_{\text {GJ }}$ must cancel the negative $\rho_{\mathrm{e}}$ to make the right-hand side be positive. That is, at the inner boundary,

$$
-\frac{\rho_{\mathrm{GJ}}}{B}=\frac{\Omega}{2 \pi c} \frac{B_{\zeta}}{B}>\frac{\left|\rho_{\mathrm{e}}\right|}{B} \sim \frac{\Omega}{2 \pi c}
$$

must be satisfied, so that the acceleration field may not change sign in the gap. It follows that the polar cap, where $B_{\zeta} \sim B$ holds, is the only place for the inner boundary of the 'outer' gap to be located, if the created particle number density in the gap is comparable to the typical Goldreich-Julian value (eq. [5]). Such a non-vacuum gap must extend from the polar cap (not from the null surface where $\rho_{\text {GJ }}$ vanishes) to the light cylinder. We can therefore conclude that the original vacuum solution obtained by CHR cannot be applied to a non-vacuum CHR picture when there is a sufficient pair production that is needed to explain the observed $\gamma$-ray luminosity.

To construct a self-consistent model, we have to solve equation (4) together with the Boltzmann equations for particles and $\gamma$-rays. In what follows, we consider this issue.

\section{Analytic Examination of the Gap Position}

Before turning to a closer examination of the particle Boltzmann equations (after $\S 4.2$ ), it is helpful to describe the gap position and width with the aid of particles continuity equations. In this section, we analytically investigate this issue.

\subsection{Particle Continuity Equations}

At time $t$, position $\boldsymbol{x}$, and momentum $\boldsymbol{p}$, the distribution function $N$ of particles obeys the following Boltzmann equation,

$$
\frac{\partial N}{\partial t}+\boldsymbol{v} \cdot \nabla N+\left(e \boldsymbol{E}+\frac{\boldsymbol{v}}{c} \times \boldsymbol{B}\right) \cdot \frac{\partial N}{\partial \boldsymbol{p}}=S(t, \boldsymbol{x}, \boldsymbol{p}),
$$

where $\boldsymbol{v} \equiv \boldsymbol{p} /\left(m_{\mathrm{e}} \Gamma\right) ; m_{\mathrm{e}}$ refers to the rest mass of an electron, and $\Gamma \equiv 1 / \sqrt{1-(|\boldsymbol{v}| / c)^{2}}$ the Lorentz factor. In a pulsar magnetosphere, the collision term $S$ consists of the terms representing the particle appearing and disappearing rates at $\boldsymbol{x}$ and $\boldsymbol{p}$ per unit time per unit phase-space volume due to pair production, pair annihilation, IC scatterings, and the synchro-curvature process. Integrating equation (7) 
over the momentum space, and assuming that $N$ vanishes rapidly enough at $p_{i} \rightarrow \pm \infty(i=1,2,3)$, we obtain

$$
\frac{\partial \tilde{N}}{\partial t}+\nabla \cdot(\langle\boldsymbol{v}\rangle \tilde{N})=\tilde{S}(t, \boldsymbol{x}, \boldsymbol{p})
$$

where the particle number density $\tilde{N}$ and the averaged particle velocity $\langle\boldsymbol{v}\rangle$ are defined by

$$
\tilde{N}(t, \boldsymbol{x}) \equiv \int_{-\infty}^{\infty} N(t, \boldsymbol{x}, \boldsymbol{p}) d^{3} \boldsymbol{p}, \quad\langle\boldsymbol{v}\rangle \equiv \frac{\int_{-\infty}^{\infty} \boldsymbol{v} N d^{3} \boldsymbol{p}}{\int_{-\infty}^{\infty} N d^{3} \boldsymbol{p}} .
$$

Since the IC scatterings and the synchro-curvature process conserve the particle number,

$$
\tilde{S}(t, \boldsymbol{x}) \equiv \int_{-\infty}^{\infty} S(t, \boldsymbol{x}, \boldsymbol{p}) d^{3} \boldsymbol{p}
$$

consists of pair production and annihilation terms. For a typical pulsar magnetosphere, the annihilation is negligibly small compared with the production. Therefore, we obtain

$\tilde{S}(t, \boldsymbol{x})=\frac{1}{c} \int_{0}^{\infty} d E_{\gamma}\left[\eta_{\mathrm{p}}\left(E_{\gamma}, \mu_{+}\right) G_{+}+\eta_{\mathrm{p}}\left(E_{\gamma}, \mu_{-}\right) G_{-}\right]$,

where $G_{+}\left(t, \boldsymbol{x}, E_{\gamma}\right)$ and $G_{-}\left(t, \boldsymbol{x}, E_{\gamma}\right)$ designate the distribution functions of outwardly and inwardly propagating $\gamma$-ray photons, respectively, having energy $E_{\gamma}$. The pair-production redistribution functions are defined by

$$
\eta_{\mathrm{p}}\left(t, \boldsymbol{x} ; E_{\gamma}, \mu\right)=c \int_{-1}^{1} d \mu(1-\mu) \int_{E_{\mathrm{th}}}^{\infty} d E_{\mathrm{x}} \frac{d N_{\mathrm{x}}}{d E_{\mathrm{x}} d \mu} \sigma_{\mathrm{p}},
$$

where $\sigma_{\mathrm{p}}\left(E_{\gamma}, E_{\mathrm{x}}, \mu\right)$ represents the pair-production cross section, and

$$
E_{\mathrm{th}} \equiv \frac{2}{1-\mu} \frac{\left(m_{\mathrm{e}} c^{2}\right)^{2}}{E_{\gamma}} ;
$$

$\cos ^{-1} \mu_{+}\left(\right.$or $\left.\cos ^{-1} \mu_{-}\right)$is the collision angle between the X-rays and the outwardly (or inwardly) propagating $\gamma$-rays.

Since the drift motion due to the gradient and the curvature of $\boldsymbol{B}$ is negligible for typical outer-gap parameters, we can decouple $\langle\boldsymbol{v}\rangle$ as

$$
\langle\boldsymbol{v}\rangle=\varpi \Omega_{\mathrm{p}} \boldsymbol{e}_{\phi}+c \cos \Phi \frac{\boldsymbol{B}_{\mathrm{p}}}{B_{\mathrm{p}}},
$$

where $\Omega_{\mathrm{p}}$ designates the angular velocity of particles due to $\boldsymbol{E}_{\perp} \times \boldsymbol{B}_{\mathrm{p}}$ drift, and $\Phi$ the projection angle of the particle three-dimensional motion onto the poloidal plane. The drift angular velocity $\Omega_{\mathrm{p}}$ coincides $\Omega$ provided that $B_{\phi}=0$ and $\left|E_{\|}\right| \ll\left|E_{\perp}\right|=$ $B_{\mathrm{p}} \varpi / \varpi_{\mathrm{LC}}$ hold. Imposing a stationary condition

$$
\frac{\partial}{\partial t}+\Omega_{\mathrm{p}} \frac{\partial}{\partial \phi}=0,
$$

assuming $B_{\phi} \ll B_{\mathrm{p}} \equiv\left|\boldsymbol{B}_{\mathrm{p}}\right|$, and utilizing $\boldsymbol{\nabla} \cdot \boldsymbol{B}=$ 0 , we obtain the following continuity equations for particles from equation (8)

$$
\pm B_{\mathrm{p}} \frac{\partial}{\partial s}\left(\frac{\tilde{N}_{ \pm}}{B_{\mathrm{p}}}\right)=\frac{1}{\lambda_{\mathrm{p}}|\cos \Phi|} \int_{0}^{\infty} d E_{\gamma}\left(G_{+}+G_{-}\right),
$$

where $B_{\mathrm{p}} \partial / \partial s=\boldsymbol{B}_{\mathrm{p}} \cdot \boldsymbol{\nabla}$. Throughout this paper, we assume $E_{\|}>0$, which does not lose any generality. In this case, for positrons (or electrons), we have $\tilde{N}=$ $\tilde{N}_{+}=\rho_{+} / e$ and $\cos \Phi=+|\cos \Phi|$ (or $\tilde{N}=\tilde{N}_{-}=$ $-\rho_{-} / e$ and $\left.\cos \Phi=-|\cos \Phi|\right)$. The pair-production mean free path $\lambda_{\mathrm{p}}(s)$ is defined by

$\frac{1}{\lambda_{\mathrm{p}}} \equiv \frac{\int_{0}^{\infty}\left[\eta_{\mathrm{p}}\left(s, E_{\gamma}, \mu_{+}\right) G_{+}+\eta_{\mathrm{p}}\left(s, E_{\gamma}, \mu_{-}\right) G_{-}\right] d E_{\gamma}}{c \int_{0}^{\infty}\left(G_{+}\left(s, E_{\gamma}\right)+G_{-}\left(s, E_{\gamma}\right)\right) d E_{\gamma}}$.

Since the number of created positrons is always equal to that of created electrons, the right-hand side of equation (16) is common for $\tilde{N}_{+}$and $\tilde{N}_{-}$. To examine the gap position, we must combine equation (16) with the $\gamma$-ray Boltzmann equations.

\subsection{Elimination of Gamma-ray Distribution Functions}

In general, the $\gamma$-ray distribution function $G_{ \pm}$at momentum $\boldsymbol{k}$ obey the following Boltzmann equations

$$
\frac{\partial G_{ \pm}}{\partial t}+c \frac{\boldsymbol{k}}{|\boldsymbol{k}|} \cdot \nabla G_{ \pm}(t, \boldsymbol{x}, \boldsymbol{k})=S_{\gamma}(t, \boldsymbol{x}, \boldsymbol{k}),
$$

where $S_{\gamma}$ represents the collision terms. Unlike the charged particles, $\gamma$-rays do not propagate along the magnetic field line at each point, because they preserve the directional information where they were emitted. However in this paper, we assume for simplicity that they propagate along the local magnetic field lines and put

$$
\frac{\boldsymbol{k}}{|\boldsymbol{k}|}=\cos \Phi_{\gamma} \frac{\boldsymbol{B}_{\mathrm{p}}}{B_{\mathrm{p}}}+\sin \Phi_{\gamma} \boldsymbol{e}_{\phi} .
$$


This assumption is justified if the magnetic field lines are nearly straight in the gap (i.e., if $W \ll \varpi_{\mathrm{LC}}$ holds). If aberration due to rotation is negligible, we obtain $\Phi_{\gamma}=0$ (or $\pi$ ) for outwardly (or inwardly) propagating $\gamma$-rays. For simplicity, we further assume that the following stationary condition is satisfied

$$
\left[\frac{\partial}{\partial t}+c \sin \Phi_{\gamma} \frac{1}{\varpi} \frac{\partial}{\partial \phi}\right] G_{ \pm}=0
$$

Then, noting that the curvature process is the dominant process in $S_{\gamma}$, compared with the IC process, the pair creation, and the pair annihilation for typical pulsar parameters, we find that $G_{ \pm}\left(s, E_{\gamma}\right)$ obey

$$
c \cos \Phi_{\gamma} B_{\mathrm{p}} \frac{\partial}{\partial s}\left(\frac{G_{ \pm}}{B_{\mathrm{p}}}\right)=\int_{1}^{\infty} d \Gamma \eta_{\mathrm{c}}\left(s, E_{\gamma}, \Gamma\right) N_{ \pm}(s, \Gamma),
$$

where (e.g., Rybicki, Lightman 1979)

$$
\begin{gathered}
\eta_{\mathrm{c}}\left(s, E_{\gamma}, \Gamma\right) \equiv \frac{\sqrt{3} e^{2} \Gamma}{h \rho_{\mathrm{c}}(s)} \frac{1}{E_{\gamma}} F\left(\frac{E_{\gamma}}{E_{\mathrm{c}}}\right), \\
E_{\mathrm{c}}(s, \Gamma) \equiv \frac{3}{4 \pi} \frac{h c \Gamma^{3}}{\rho_{\mathrm{c}}}, \\
F(x) \equiv x \int_{x}^{\infty} K_{\frac{5}{3}}(t) d t ;
\end{gathered}
$$

$\rho_{\mathrm{c}}(s)$ is the curvature radius of the magnetic field lines and $K_{5 / 3}$ is the modified Bessel function of $5 / 3$ order. The effect of the broad spectrum of curvature $\gamma$-rays is represented by the factor $F\left(E_{\gamma} / E_{\mathrm{c}}\right)$ in equation (22).

Integrating equation (21) over $E_{\gamma}$, combining with equations (16), and assuming $\partial_{s}\left(\lambda_{\mathrm{p}} \cos \Phi\right)=0$, we obtain

$\pm \frac{d^{2}}{d s^{2}}\left(\frac{N_{ \pm}}{B_{\mathrm{p}}}\right)=\frac{1}{\left|\cos \Phi \cos \Phi_{\gamma}\right|} \frac{1}{\lambda_{\mathrm{p}} c} \frac{N_{+}-N_{-}}{B_{\mathrm{p}}} \int_{0}^{\infty} \eta_{\mathrm{c}} d E_{\gamma}$,

where $0<\Phi_{\gamma}<\pi / 2$ (or $\pi / 2<\Phi_{\gamma}<\pi$ ) is applied to outwardly (or inwardly) propagating $\gamma$-rays.

\subsection{Real Charge Density in the Gap}

One combination of the two independent equations (25) yields the current conservation law; that is, the total current density per magnetic flux tube,

$$
j_{\text {tot }}=\frac{2 \pi c e}{\Omega} \frac{\tilde{N}_{+}(s)+\tilde{N}_{-}(s)}{B_{\mathrm{p}}(s)}
$$

is conserved along the field lines. (Note that it can be derived directly from eq. [16].) Another combination gives

$$
\frac{d^{2}}{d s^{2}}\left(\frac{\tilde{N}_{+}-\tilde{N}_{-}}{B_{\mathrm{p}}}\right)=\frac{2}{W} \cdot \frac{N_{\gamma}}{\lambda_{\mathrm{p}}\left|\cos \Phi_{\gamma}\right|} \frac{\tilde{N}_{+}-\tilde{N}_{-}}{B_{\mathrm{p}}},
$$

where

$$
N_{\gamma} \equiv \frac{W}{c|\cos \Phi|} \int_{0}^{\infty} \eta_{\mathrm{c}}\left(s, \Gamma, E_{\gamma}\right) d E_{\gamma}
$$

refers to the expectation value of the number of $\gamma$ rays emitted by a single particle that runs the gap width, $W$. Lorentz factor appearing in $\eta_{\mathrm{c}}$ should be evaluated at each position $s$.

Exactly speaking, $\lambda_{\mathrm{p}}$ depends on $G_{+}$and $G_{-}$; thus, the $\gamma$-ray distribution functions are not eliminated in equation (25). Nevertheless, for analytic (and qualitative) discussion of the gap position, we may ignore such details and adopt equation (27).

A typical $\gamma$-ray propagates the length $W /\left(2\left|\cos \Phi_{\gamma}\right|\right)$ within the gap that is transversely thick. Thus, so that a stationary pair-production cascade may be maintained, the optical depth, $W /\left(2\left|\cos \Phi_{\gamma}\right| \lambda_{\mathrm{p}}\right)$, must equal the expectation value for a $\gamma$-ray to materialize with the gap, $N_{\gamma}^{-1}$. We thus obtain the following condition: $W / 2=\left|\cos \Phi_{\gamma}\right| \lambda_{\mathrm{p}} / N_{\gamma}$. This relation holds for a self-sustaining gap in which all the particles are supplied by the pair production. If there is an external particle injection, the injected particles also contribute for the $\gamma$-ray emission. As a result, a stationary gap can be maintain with a smaller width compared to the case of no particle injection. Taking account of such injected particles, we can constrain the half gap width as

$$
\frac{W}{2}=\frac{\lambda_{\mathrm{p}}\left|\cos \Phi_{\gamma}\right|}{N_{\gamma}} \cdot \frac{j_{\text {gap }}}{j_{\text {tot }}},
$$

where $j_{\text {gap }}$ and $j_{\text {tot }}$ refer to the created and total current densities per unit magnetic flux tube. Equation (29) is automatically satisfied if we solve the set of Maxwell and stationary Boltzmann equations. Here, $j_{\text {gap }}$ is related with the particle injection rate across the boundaries as follows:

$$
\begin{aligned}
\frac{\Omega}{2 \pi c e} j_{\text {gap }} & =\frac{\tilde{N}_{+}\left(s^{\text {out }}\right)}{B_{\mathrm{p}}\left(s^{\text {out }}\right)}-\frac{\tilde{N}_{+}\left(s^{\text {in }}\right)}{B_{\mathrm{p}}\left(s^{\text {in }}\right)} \\
& =\frac{\tilde{N}_{-}\left(s^{\text {in }}\right)}{B_{\mathrm{p}}\left(s^{\text {in }}\right)}-\frac{\tilde{N}_{-}\left(s^{\text {out }}\right)}{B_{\mathrm{p}}\left(s^{\text {out }}\right)},
\end{aligned}
$$

where $s^{\text {in }}$ and $s^{\text {out }}$ designate the position of the inner and the outer boundaries, respectively. That is, $W=$ $s^{\text {out }}-s^{\text {in }}$. 
With the aid of identity (29), we can rewrite equation (27) into the form

$$
\frac{d^{2}}{d s^{2}}\left(\frac{\tilde{N}_{+}-\tilde{N}_{-}}{B_{\mathrm{p}}}\right)=\frac{j_{\text {gap }}}{j_{\text {tot }}} \frac{4}{W^{2}} \frac{\tilde{N}_{+}-\tilde{N}_{-}}{B_{\mathrm{p}}} .
$$

To solve this differential equation, we impose the following two boundary conditions:

$$
\begin{gathered}
c e \frac{\tilde{N}_{+}\left(s^{\text {in }}\right)}{B_{\mathrm{p}}\left(s^{\text {in }}\right)}=\frac{\Omega}{2 \pi} j^{\text {in }}, \\
c e \frac{\tilde{N}_{-}\left(s^{\text {out }}\right)}{B_{\mathrm{p}}\left(s^{\text {out }}\right)}=\frac{\Omega}{2 \pi} j^{\text {out }} .
\end{gathered}
$$

Then, equation (30), (32), and (33) give

$$
\frac{\tilde{N}_{+}-\tilde{N}_{-}}{B_{\mathrm{p}}}=-\frac{\Omega}{2 \pi c e}\left(j_{\text {gap }}-j^{\text {in }}+j^{\text {out }}\right)
$$

at $s=s^{\text {in }}$, and

$$
\frac{\tilde{N}_{+}-\tilde{N}_{-}}{B_{\mathrm{p}}}=\frac{\Omega}{2 \pi c e}\left(j_{\text {gap }}+j^{\text {in }}-j^{\text {out }}\right)
$$

at $s=s^{\text {out }}$. Under boundary conditions (34) and (35), equation (31) is solved as

$$
\begin{gathered}
\frac{\tilde{N}_{+}-\tilde{N}_{-}}{B_{\mathrm{p}}}=\frac{\Omega}{2 \pi c e}\left[j_{\text {gap }} \frac{\sinh \left(\sqrt{\frac{j_{\text {gap }}}{j_{\text {tot }}}} \frac{s-s_{\text {cnt }}}{W / 2}\right)}{\sinh \left(\sqrt{\frac{j_{\text {gap }}}{j_{\text {tot }}}}\right)}\right. \\
\left.+\left(j^{\text {in }}-j^{\text {out }}\right) \frac{\cosh \left(\sqrt{\frac{j_{\text {gap }}}{j_{\text {tot }}}} \frac{s-s_{\text {cnt }}}{W / 2}\right)}{\cosh \left(\sqrt{\frac{j_{\text {gap }}}{j_{\text {tot }}}}\right)}\right],
\end{gathered}
$$

where the gap center position is defined by

$$
s_{\mathrm{cnt}} \equiv \frac{s^{\text {in }}+s^{\text {out }}}{2} .
$$

Note that $e\left(\tilde{N}_{+}-\tilde{N}_{-}\right)=\rho_{\mathrm{e}}$ represents the real charge density, which appears in the Poisson equation. Thus, substituting equation (36) into (4), we obtain

$$
\begin{aligned}
-\nabla^{2} \Psi & =\frac{2 B_{\mathrm{p}} \Omega}{c}\left[j_{\text {gap }} f_{\text {odd }}\left(\frac{s-s_{\mathrm{cnt}}}{W / 2}\right)\right. \\
+ & \left.\left(j^{\text {in }}-j^{\text {out }}\right) f_{\text {even }}\left(\frac{s-s_{\text {cnt }}}{W / 2}\right)+\frac{B_{\zeta}}{B}\right],
\end{aligned}
$$

where

$$
\begin{gathered}
f_{\text {odd }}(x) \equiv \frac{\sinh \left(x \sqrt{j_{\text {gap }} / j_{\text {tot }}}\right)}{\sinh \left(\sqrt{j_{\text {gap }} / j_{\text {tot }}}\right)}, \\
f_{\text {even }}(x) \equiv \frac{\cosh \left(x \sqrt{j_{\text {gap }} / j_{\text {tot }}}\right)}{\cosh \left(\sqrt{j_{\text {gap }} / j_{\text {tot }}}\right)} .
\end{gathered}
$$

At the inner boundary, $s=s^{\text {in }},\left(s-s_{\text {cnt }}\right) /(W / 2)=$ -1 holds; therefore, we obtain

$$
-\nabla^{2} \Psi=\frac{2 B_{\mathrm{p}} \Omega}{c}\left(-j_{\text {gap }}+j^{\text {in }}-j^{\text {out }}+\frac{B_{\zeta}}{B_{\mathrm{p}}}\right) .
$$

In the CHR picture, it is assumed that there is no particle injection across either of the boundaries (i.e., $\left.j^{\text {in }}=j^{\text {out }}=0\right)$ and that the current density associated with the created particles becomes of the order of the typical Goldreich-Julian value (i.e., $j_{\text {gap }} \sim 1$ ). It follows that the inner boundary of an 'outer gap' should be located close to the star, where $B_{\zeta} \sim B_{\mathrm{p}}$ holds; this conclusion is consistent with what was obtained in $\S 2$.

\subsection{Gap Position vs. Particle Injection}

To examine the Poisson equation (38) analytically, we assume that the transfield thickness of the gap is greater than $W$ and replace $\nabla^{2} \Psi$ with $d^{2} \Psi / d s^{2}$. Furthermore, we neglect the current created in the gap and put $j_{\text {gap }} \sim 0$.

First, consider the case when particles are injected across neither of the boundaries(i.e., $j^{\text {in }}=j^{\text {out }}=0$ ). It follows that the derivative of the $E_{\|}$vanishes at the null surface, where $B_{\zeta}$ vanishes. We may notice that $-d^{2} \Psi / d s^{2}=d E_{\|} / d s$ is positive at the inner part of the gap and becomes negative at the outer part. The acceleration field is screened out at the boundaries by virtue of the spatial distribution of the local Goldreich-Julian charge density, $\rho_{\mathrm{GJ}}$. Therefore, we can conclude that the gap is located (or centers) around the null surface, if there is no particle injection from outside. This conclusion can be easily generalized to the case $j^{\text {in }}=j^{\text {out }} \neq 0$.

Secondly, consider the case when particles are injected across the inner boundary at $s=s^{\text {in }}$ (or in general, when $j^{\text {in }}-j^{\text {out }}>0$ holds). Since the function $f_{\text {even }}$ is positive at arbitrary $s$, the gap center is located at a place where $B_{\zeta}$ is negative, that is, outside of the null surface. In particular, when $j^{\text {in }}-j^{\text {out }} \sim 1$ holds, $d E_{\|} / d s$ vanishes at the place where $B_{\zeta} \sim-B$ 
holds. In a vacuum, static dipole field, $B_{\zeta} \sim-B$ is realized along the last-open field line near to the light cylinder. Therefore, the gap should be located close to the light cylinder, if the injected particle flux across the inner boundary approaches the typical GoldreichJulian value. We may notice here that $f_{\text {even }}$ is less than unity, because $\left|s-s_{\text {cnt }}\right|$ does not exceed $W / 2$.

Thirdly and finally, consider the case when $j^{\text {in }}-$ $j^{\text {out }} \sim-1$ holds. In this case, $d E_{\|} / d s$ vanishes at the place where $B_{\zeta} \sim B$. Therefore, an 'outer' gap should be located in the polar cap, if a Goldreich-Julian particle flux is injected across the outer boundary.

\section{The Set of Maxwell and Boltzmann Equa- tions}

To examine the gap electrodynamics more quantitatively, we have to solve numerically the Poisson equation for the electrostatic potential together with the Boltzmann equations for particles and $\gamma$-rays. To this aim, we reduce these equations to a tractable forms in this section.

\subsection{One-dimensional Poisson Equation}

For simplicity, we assume that a gap is transversely thick in the sense $D_{\perp}>W$ (or at least $D_{\perp} \sim W$ ). In this case, the derivative with respect to $z$ in the lefthand side of the Poisson equation (4) can be approximated with $-\Psi / D_{\perp}^{2}$. Thus, we obtain the following one-dimensional expression of the Poisson equation (4):

$$
-\frac{\partial^{2} \Psi}{\partial s^{2}}=-\frac{\Psi}{D_{\perp}^{2}}+4 \pi\left[\rho_{\mathrm{e}}(s)+\frac{\Omega B_{\zeta}(s)}{2 \pi c}\right],
$$

where

$$
\rho_{\mathrm{e}}(s)=e \int_{1}^{\infty} d \Gamma\left[N_{+}(s, \Gamma)-N_{-}(s, \Gamma)\right] .
$$

The particle distribution functions $N_{+}$and $N_{-}$obey the Boltzmann equations that will be described just below.

\subsection{Particle Boltzmann Equations}

In this section, we consider the Boltzmann equations (7), which is necessary to investigate energy distribution of particles. It should be noted that quantum effects can be neglected in the outer magnetosphere, because the magnetic field is much less than the critical value $\left(4.41 \times 10^{13} \mathrm{G}\right)$. As a result, synchrocurvature radiation takes place continuously and can be regarded as an external force acting on a particle. If we instead put the collision term associated with the synchro-curvature process in the right-hand side, the energy transfer in each collision would be too small to be resolved by the energy grids.

In the same manner as we derived the stationary continuity equations (16), we impose the stationary condition (15). Then, neglecting the pitch-angle dependence of the particle distribution functions, and approximating the collision term associated with the curvature process as an external force acting on a particle, we obtain (Appendix A in Paper X)

$B_{\mathrm{p}} \frac{\partial}{\partial s}\left(\frac{N_{+}}{B_{\mathrm{p}}}\right)+\left[e E_{\|}-\frac{P_{\mathrm{CV}}(s, \Gamma)}{c}\right] \frac{\partial N_{+}}{\partial \Gamma}=S_{+}(s, \Gamma)$,

$B_{\mathrm{p}} \frac{\partial}{\partial s}\left(\frac{N_{-}}{B_{\mathrm{p}}}\right)-\left[e E_{\|}-\frac{P_{\mathrm{CV}}(s, \Gamma)}{c}\right] \frac{\partial N_{-}}{\partial \Gamma}=-S_{-}(s, \Gamma)$,

where the radiation-reaction force is given by

$$
\frac{P_{\mathrm{CV}}(s, \Gamma)}{c}=\frac{2 e^{2} \Gamma^{4}}{3 \rho_{\mathrm{c}}^{2}(s)}
$$

$\rho_{\mathrm{c}}$ is the curvature radius of the magnetic field line.

\subsubsection{Collision terms}

We assume in this paper that $\gamma$-rays are either outwardly or inwardly propagating along the local magnetic field lines. We also assume that the soft photons are emitted from the neutron star and hence unidirectional at the gap. Then the cosine of the collision angle $\mu$ has a unique value $\mu_{+}$or $\mu_{-}$, for outwardly or inwardly propagating $\gamma$-rays, respectively; $\mu_{+}$and $\mu_{-}$are determined by the magnetic inclination $\alpha_{\mathrm{i}}$ at each position $s$ (eq. [23] in Paper VII). Under these assumptions, the source term can be expressed as

$$
\begin{aligned}
& S_{+}(s, \Gamma)=-\int_{E_{\gamma}<\Gamma} d E_{\gamma} \eta_{\mathrm{IC}}^{\gamma}\left(E_{\gamma}, \Gamma, \mu_{+}\right) N_{+}(s, \Gamma) \\
& \quad+\int_{\Gamma_{i}>\Gamma} d \Gamma_{i} \eta_{\mathrm{IC}}^{\mathrm{e}}\left(\Gamma_{i}, \Gamma, \mu_{+}\right) N_{+}\left(s, \Gamma_{i}\right)+Q_{\mathrm{p}}(s, \Gamma),
\end{aligned}
$$

$$
\begin{aligned}
& S_{-}(s, \Gamma)=-\int_{E_{\gamma}<\Gamma} d E_{\gamma} \eta_{\mathrm{IC}}^{\gamma}\left(E_{\gamma}, \Gamma, \mu_{-}\right) N_{-}(s, \Gamma) \\
& +\int_{\Gamma_{i}>\Gamma} d \Gamma_{i} \eta_{\mathrm{IC}}^{\mathrm{e}}\left(\Gamma_{i}, \Gamma, \mu_{-}\right) N_{-}\left(s, \Gamma_{i}\right)+Q_{\mathrm{p}}(s, \Gamma),
\end{aligned}
$$


where the pair-production rate per unit volume per unit Lorentz factor is defined as

$Q_{\mathrm{p}} \equiv \int d E_{\gamma}\left[\frac{\partial \eta_{\mathrm{p}}\left(E_{\gamma}, \Gamma, \mu_{+}\right)}{\partial \Gamma} G_{+}+\frac{\partial \eta_{\mathrm{p}}\left(E_{\gamma}, \Gamma, \mu_{-}\right)}{\partial \Gamma} G_{-}\right]$.

The positrons (or electrons) are supposed to collide with the soft photons at the same angle as the outwardly (or inwardly) propagating $\gamma$-rays; therefore, the same collision angle $\cos ^{-1} \mu_{+}\left(\right.$or $\left.\cos ^{-1} \mu_{-}\right)$is used for both IC scatterings and pair production in equations (47)-(49). The collisions tend to be head-on (or tail-on) for inwardly (or outwardly) propagating $\gamma$-rays, as the gap approaches the star. The pairproduction redistribution function is given by

$$
\frac{\partial \eta_{\mathrm{p}}}{\partial \Gamma}\left(E_{\gamma}, \Gamma, \mu_{ \pm}\right)=\left(1-\mu_{ \pm}\right) \int_{E_{\mathrm{th}}}^{\infty} d E_{\mathrm{s}} \frac{d F_{\mathrm{s}}}{d E_{\mathrm{s}}} \frac{d \sigma_{\mathrm{p}}}{d \Gamma},
$$

where the pair-production threshold energy is defined by equation (13). The differential cross section $d \sigma_{\mathrm{p}} / d \Gamma$ is given in numerous textbooks in quantum electrodynamics (e.g., Akhiezer \& Berestetskii 1965; or eq. [32] in Paper X)

The IC redistribution function $\eta_{\mathrm{IC}}^{\gamma}\left(E_{\gamma}, \Gamma, \mu\right)$ represents the probability that a particle with Lorentz factor $\Gamma$ upscatters photons into energies between $E_{\gamma}$ and $E_{\gamma}+d E_{\gamma}$ per unit time when the collision angle is $\cos ^{-1} \mu$. On the other hand, $\eta_{\mathrm{IC}}^{\mathrm{e}}\left(\Gamma_{i}, \Gamma, \mu\right)$ describes the probability that a particle changes Lorentz factor from $\Gamma_{i}$ to $\Gamma$ in a scattering. Thus, energy conservation gives

$$
\eta_{\mathrm{IC}}^{\mathrm{e}}\left(\Gamma_{i}, \Gamma_{f}, \mu\right)=\eta_{\mathrm{IC}}^{\gamma}\left[m_{\mathrm{e}} c^{2}\left(\Gamma_{i}-\Gamma_{f}\right), \Gamma_{i}, \mu\right]
$$

In general, $\eta_{\mathrm{IC}}^{\gamma}$ is defined by the soft photon flux $d F_{\mathrm{s}} / d \epsilon_{\mathrm{s}}$ and the Klein-Nishina cross section $\sigma_{\mathrm{KN}}$ as follows:

$$
\begin{aligned}
& \eta_{\mathrm{IC}}^{\gamma}\left(E_{\gamma}, \Gamma, \mu_{ \pm}\right)=\left(1-\beta \mu_{ \pm}\right) \\
& \quad \times \quad \int_{0}^{\infty} d E_{\mathrm{s}} \frac{d F_{\mathrm{s}}}{d E_{\mathrm{s}}} \int_{-1}^{1} d \Omega_{\gamma}^{*} \frac{d \sigma_{\mathrm{KN}}^{*}}{d E_{\gamma}{ }^{*} d \Omega_{\gamma}^{*}} \frac{d E_{\gamma}{ }^{*}}{d E_{\gamma}}
\end{aligned}
$$

where $\beta \equiv \sqrt{1-1 / \Gamma^{2}}$ is virtually unity, $\Omega_{\gamma}$ the solid angle of upscattered photon, the asterisk denotes the quantities in the electron (or positron) rest frame. In the rest frame of a particle, a scattering always takes place well above the resonance energy. Thus, the classical formula of the Klein-Nishina cross section can be applied to the present problem. The soft photon flux per unit photon energy $E_{\mathrm{s}}\left[s^{-1} \mathrm{~cm}^{-2} \mathrm{ergs}^{-1}\right]$ is written as $d F_{\mathrm{s}} / d E_{\mathrm{s}}$. To obtain $\eta_{\mathrm{IC}}^{\gamma}$ 's for individual pulsars, we substitute the observed X-ray spectrum $d F_{\mathrm{s}} / d E_{\mathrm{s}}$ and execute integration over $E_{\mathrm{s}}$ and $\Omega_{\gamma}^{*}$. For further details of $\eta_{\mathrm{IC}}^{\gamma}$, see Appendix B in Paper X.

\subsection{Gamma-ray Boltzmann Equations}

Let us briefly comment on the $\gamma$-ray Boltzmann equations. We recover $\gamma$-ray production due to IC scatterings and absorption due to pair production in the right-hand side of equation (21) to obtain

$$
\begin{gathered}
\pm c\left|\cos \Phi_{\gamma}\right| B_{\mathrm{p}} \frac{\partial}{\partial s}\left(\frac{G_{ \pm}}{B_{\mathrm{p}}}\right)=-\int d \Gamma \frac{\partial \eta_{\mathrm{p}}}{\partial \Gamma} \cdot G_{ \pm}\left(s, E_{\gamma}\right) \\
+\quad \int_{1}^{\infty} d \Gamma\left[\eta_{\mathrm{IC}}^{\gamma}\left(E_{\gamma}, \Gamma, \mu_{ \pm}\right)+\eta_{\mathrm{c}}\left(E_{\gamma}, \Gamma\right)\right] N_{ \pm}(s, \Gamma) .
\end{gathered}
$$

We integrate both sides of equation (53) over $E_{\gamma}$ in appropriate energy bins to reduce them into ordinary differential equations.

In short, the set of Maxwell and Botzmann equations consist of equations (42), (44), (45), and (53). The Poisson equation and the $\gamma$-ray Boltzmann equations become ordinary differential equations, which can be straightforwardly solved by a simple discretization. On the other hand, the hyperbolic-type partial differential equations (44) and (45) are solved by the Cubic Interpolated Propagation (CIP) scheme (e.g., Yabe \& Aoki 1991, Yabe, Xiao, \& Utsumi 2001).

\subsection{Boundary Conditions}

In this section, we consider the boundary conditions to solve the set of Maxwell and Boltzmann equations. Diving the $\gamma$-ray energies into $m$ bins, and diving the Lorentz factors into $n$ bins, we impose the following boundary conditions at the inner (starward) boundary $\left(s=s^{\text {in }}\right)$

$$
\begin{gathered}
E_{\|}\left(s^{\mathrm{in}}\right)=0, \quad \psi\left(s^{\mathrm{in}}\right)=0 \\
\int_{b_{i}}^{b_{i+1}} G_{+}\left(s^{\mathrm{in}}, E_{\gamma}\right) d E_{\gamma}=0 \quad \text { for } \quad i=1,2, \ldots m
\end{gathered}
$$

and

$$
N_{+}\left(s^{\mathrm{in}}, \Gamma_{j}\right)=\frac{\Omega B_{\mathrm{p}}\left(s^{\mathrm{in}}\right)}{2 \pi c e} y\left(\Gamma_{j}\right) \quad \text { for } \quad j=1,2, \ldots n,
$$


where $y$ is an appropriate function satisfying $\int_{1}^{\infty} y(\Gamma) d \Gamma=$ $j^{\text {in }}$. Moreover, current conservation law (26) gives

$$
\int_{1}^{\infty} N_{-}\left(s^{\text {in }}, \Gamma\right) d \Gamma=\frac{\Omega B_{\mathrm{p}}\left(s^{\text {in }}\right)}{2 \pi c e}\left(j_{\text {tot }}-j^{\text {in }}\right) .
$$

At the outer boundary $\left(s=s^{\text {out }}\right)$, we impose

$$
\begin{gathered}
E_{\|}\left(s^{\text {out }}\right)=0, \\
\int_{b_{i}}^{b_{i+1}} G_{-}\left(s^{\text {out }}, E_{\gamma}\right) d E_{\gamma}=0 \text { for } i=1,2, \ldots m \\
N_{-}\left(s^{\text {out }}, \Gamma_{j}\right)=\frac{\Omega B_{\mathrm{p}}\left(s^{\text {out }}\right)}{2 \pi c e} y\left(\Gamma_{j}\right) \text { for } j=1,2, \ldots n .
\end{gathered}
$$

The current density created in the gap per unit flux tube can be expressed as

$$
j_{\text {gap }}=j_{\text {tot }}-j^{\text {in }}-j^{\text {out }} .
$$

We adopt $j_{\text {gap }}, j^{\text {in }}$, and $j^{\text {out }}$ as the free parameters. We chose $y(\Gamma)$ so that the initial spectrum of the injected particles may peak near the lowest energy bin (e.g., solid curve in fig. 4); then, the results little depend on the detailed form of $y(\Gamma)$.

We have totally $2 m+2 n+4$ boundary conditions (54)-(60) for $2 m+2 n+2$ unknown functions

$\int_{b_{i}}^{b_{i+1}} G_{ \pm}\left(s, E_{\gamma}\right) d E_{\gamma}, N_{ \pm}\left(s, \Gamma_{j}\right), \Psi(s)$, and $E_{\|}(s)$. Therefore, two extra boundary conditions must be compensated by making the positions of the boundaries $s^{\text {in }}$ and $s^{\text {out }}$ be free. The two free boundaries appear because $E_{\|}=0$ is imposed at both the boundaries and because $j_{\text {gap }}$ is externally imposed. In other words, the gap boundaries $\left(s^{\text {in }}\right.$ and $\left.s^{\text {out }}\right)$ shift, if $j^{\text {in }}$ and/or $j^{\text {out }}$ varies. That is, the gap position, as well as its width $W$, cannot be artificially hypothesized as in previous outer-gap models. They should be selfconsistently solved from the set of Maxwell and Boltzmann equations.

\section{Application to Individual Pulsars}

To solve the set of the Maxwell and Boltzmann equations, we must specify the X-ray field, $d F_{\mathrm{S}} / d \epsilon_{\mathrm{s}}$, which is necessary to compute the pair-production redistribution function (eq. [50]). In this paper, we use the X-ray fluxes and spectra observed for individual rotation-powered pulsars. In $\S 5.1$, we summarize the observed properties of the X-ray field. Then we apply the theory to the Vela pulsar in $\S 5.2$, to PSR B170644 in $\S 5.3$, to the Geminga pulsar in $\S 5.4$, and to
PSR B1055-52 in $\S 5.5$. We assume that the solid angle of the emitted $\gamma$-rays is 1 ster throughout this paper.

\subsection{Input Soft Photon field}

We consider the photons emitted from the neutron star surface as the seed photons for $(\gamma-\gamma)$ pairproduction and IC scatterings. That is, we do not consider power-law X-ray components, because they are probably magnetospheric and beamed away from the accelerator. We evaluate the IR photon field, which is needed to compute the IC scattering rate, from the Rayleigh-Jeans tail of the surface thermal component. In table 1, we present the observed properties of the four $\gamma$-ray pulsars exhibiting surface Xray components, in order of spin-down luminosity, $L_{\text {spin }}$.

Vela (J0835-4513) From Chandra observations in $0.25-8.0 \mathrm{keV}$, the spectrum of this pulsar is turned out to consist of two distinct component: A soft, thermal component and a hard, power-law component. As stated just above, we consider only the former component as the X-ray field illuminating the outer gap. This component can be modeled as a magnetic hydrogen atmosphere spectrum with effective temperature $k T=0.68 \mathrm{MK}$ (Pavlov et al. 2001). Based on high-resolution Ca II and Na I absorption-line spectra toward $68 \mathrm{OB}$ stars in the direction of the Vela supernova remnant, Cha, Sembach, and Danks (1999) determined the distance to be $250 \pm 30 \mathrm{pc}$.

B1706-44 (J1710-4432) Gotthelf, Halpern, Dodson (2002) reported a broad, single-peaked pulsed profile with pulsed fraction of $23 \%$, using the High Resolution Camera on-board the Chandra X-ray observatory. They fitted the spectroscopic data to find (at least) two components: A blackbody of $k T=143 \mathrm{eV}$ with $A=0.129 A_{*}$ and a power-law component with photon index of -2.0 , where $A_{*}=4 \pi(10 \mathrm{~km})^{2}$. We consider that the former component illuminates the gap efficiently and neglect the latter one. We adopt $d=2.5 \mathrm{kpc}$ as a compromise between the smaller dispersion-measure distance of $1.8 \mathrm{kpc}$ based on the free electron model by Taylor and Cordes (1993) and the larger H I kinematic distance of $2.4-3.2 \mathrm{kpc}$ derived by Koribalski et al. (1995).

Geminga (J0633+1746) The X-ray spectrum consists of two components: the soft surface blackbody with $k T_{\mathrm{s}}=50 \mathrm{eV}$ and $A_{\mathrm{s}}=0.21 A_{*}(d / 0.16)^{2}$ and a hard power law with $\alpha=-1.6$ (Halpern \& Wang 1997). A parallax distance of $160 \mathrm{pc}$ was estimated 
Table 1: Input thermal X-ray field

\begin{tabular}{lcccrrcccll}
\hline \hline pulsar & $\begin{array}{c}\lg L_{\text {spin }} \\
\mathrm{ergs} \mathrm{s}^{-1}\end{array}$ & $\begin{array}{c}\text { distance } \\
\mathrm{kpc}\end{array}$ & $\begin{array}{c}\Omega \\
\mathrm{rad} \mathrm{s}^{-1}\end{array}$ & $\begin{array}{c}\lg B_{\mathrm{s}} \\
\mathrm{G}\end{array}$ & $\begin{array}{r}k T_{\mathrm{s}} \\
\mathrm{eV}\end{array}$ & $\begin{array}{c}A_{\mathrm{s}} / A_{*}^{\dagger}{ }^{\dagger} \\
k T_{\mathrm{h}} \\
\mathrm{eV}\end{array}$ & $A_{\mathrm{h}} / A_{*}^{\dagger}{ }^{\dagger}$ & model & refs. \\
\hline Vela & 36.84 & 0.25 & 70.4 & 12.53 & 59 & 1.000 & $\ldots$ & $\ldots$ & hydrogen atm. & 1 \\
B1706-44 & 36.53 & 2.50 & 61.3 & 12.49 & 143 & 0.129 & $\ldots$ & $\ldots$ & blackbody & 2 \\
Geminga & 34.51 & 0.16 & 26.5 & 12.21 & 50 & 0.208 & $\ldots$ & $\ldots$ & blackbody & 3,4 \\
B1055-52 & 34.48 & 1.53 & 31.9 & 12.03 & 78 & 1.340 & 740 & $10^{-5.32}$ & blackbody & 5 \\
\hline
\end{tabular}

$\dagger A_{*}=4 \pi(10 \mathrm{~km})^{2} ; \quad$ 1: Pavlov et al. 2001; 2: Gotthelf, Halpern, Dodson 2002; 3 : Halpern \& Wang 1997; 4: Becker \& Trümper 1996; 5: Mineo et al. 2002.

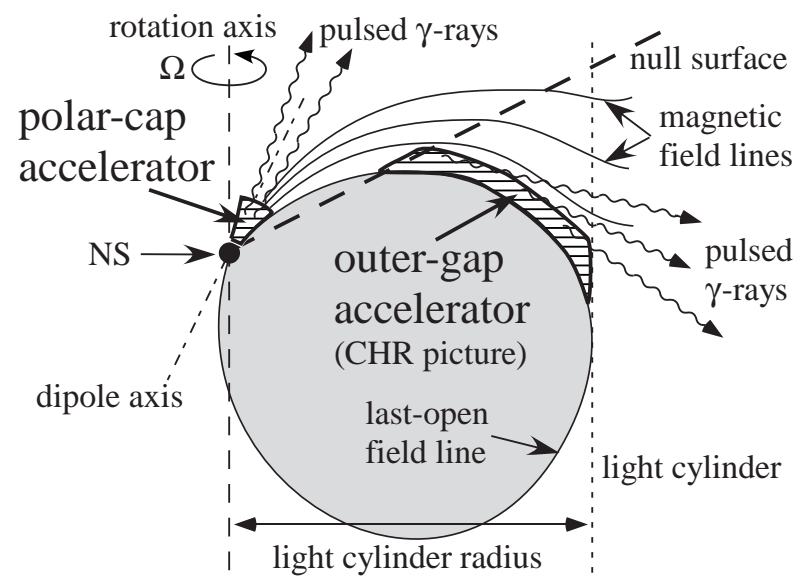

Fig. 1.- Schematic picture (side view) of the two representative models for accelerators (hatched regions) in a pulsar magnetosphere. The small filled circle indicated by 'NS' represents the neutron star, which is rotating around the rotation axis (light dashed line). The light cylinder (dotted line) is located typically a few hundred neutron-star radii from the rotation axis. On the null surface (heavy dashed line), the magnetic field component projected along the rotation axis, vanishes. The closed field lines, which do not penetrate the light cylinder, are in the shaded region. It is hypothesized in the CHR picture that the outer-gap accelerator extends between the null surface and the light cylinder. from HST observations (Caraveo et al. 1996).

B1055-52 (J1059-5237) Analyzing BeppoSAX data, Mineo et al. (2002) reported that the X-ray spectrum consists of two components: a soft blackbody with $k T_{\mathrm{s}}=78 \mathrm{eV}$ and $A_{\mathrm{s}}=1.3 A_{*}(d / 1.53)^{2}$ and a hard blackbody with $k T_{\mathrm{h}}=740 \mathrm{eV}$ and $A_{\mathrm{h}}=4.8 \times$ $10^{-6}(d / 1.53)^{2}$. They pointed out that a blackbody+ powerlaw model also fits the data. However, the results differ little between the two models, by virtue of the negative feedback effect of the gap electrodynamics (§ 6.1). Thus, we adopt the former, two-blackbody model. The distance is estimated to be $1.53 \mathrm{kpc}$ from dispersion measure (Taylor \& Cordes 1993).

\subsection{The Vela pulsar}

\subsubsection{Acceleration Field and Characteristics}

We apply the theory to the Vela pulsar. Let us first consider the spatial distribution of $E_{\|}$. For this pulsar, a small created current density $j_{\text {gap }}=4.6 \times 10^{-5}$ gives the best-fit spectrum (see $\S 5.2 .3$ for details).

To compare the effects of particle injection, we present the $E_{\|}$distribution for the three cases of $j^{\text {in }}=0$ (solid), 0.25 (dashed), and 0.50 (dash-dotted) in figure 2. The magnetic inclination is chosen to be $\alpha_{\mathrm{i}}=75^{\circ}$. We adopt $j^{\text {out }}=0$ throughout this paper, unless its value is explicitly specified.

As the solid line shows, the gap is located around the null surface when there is no particle injection across either of the boundaries. Moreover, $E_{\|}$varies quadratically, because the Goldreich-Julian charge density deviates from zero linearly near to the null surface.

As the dashed and dash-dotted lines indicate, the gap shifts outwards as $j^{\text {in }}$ increases. When $j^{\text {in }}=0.5$ for instance, the gap is located on the half way between the null surface and the light cylinder. This 
result is consistent with the analytic prediction given in $\S 3.4$. The gap width increases as it shifts outwards, because $\lambda_{\mathrm{p}}$ in equation (29) increases due to decreased X-ray density at large distances from the star.

In figure 3 , we present the characteristics of partial differential equation (44) for positrons by solid lines, together with $E_{\|}(s)$ when $j^{\text {in }}=0.25$ and $\alpha_{\mathrm{i}}=75^{\circ}$ (i.e., the dashed line in fig. 2). We also superpose the equilibrium Lorentz factor that would be obtained if we assumed the balance between the curvature radiation reaction and the electrostatic acceleration, as the dotted line. It follows that the particles are not saturated at the equilibrium Lorentz factor in most portions of the gap.

In the outer part of the gap where $E_{\|}$is decreasing, characteristics begin to concentrate; as a result, the energy distribution of outwardly propagating particles forms a 'shock' in the Lorentz factor direction. However, the particle Lorentz factors do not match the equilibrium value (dotted line). For example, near the outer boundary, the particles have larger Lorentz factors compared with the equilibrium value, because the curvature cooling scale is longer than the gap width. Thus, we must discard the mono-energetic approximation that all the particles migrate at the equilibrium Lorentz factor as adopted in Papers I through IX. We instead have to solve the energy dependence of the particle distribution functions explicitly.

The particles emit $\gamma$-rays not only inside of the gap but also outside of it, being decelerated by the curvature radiation-reaction force. The length scale of the deceleration is given by

$$
\begin{aligned}
l_{\text {curv }} & =c \cdot \frac{\Gamma m_{\mathrm{e}} c^{2}}{\frac{2 e^{2}}{3 c^{3}} \Gamma^{4}\left(\frac{c^{2}}{\rho_{\mathrm{c}}}\right)^{2}} \\
& =0.4 \varpi_{\mathrm{LC}} \Omega_{2}{ }^{-1}\left(\frac{\Gamma}{10^{7}}\right)^{-3}\left(\frac{\rho_{\mathrm{c}}}{\varpi_{\mathrm{LC}} / 2}\right)^{2}
\end{aligned}
$$

Since the typical Lorentz factor is a few times of $10^{7}$, $l_{\text {curv }}$ is typically much less than $\varpi_{\mathrm{LC}}$. Therefore, the escaping particles lose most of their energies well inside of the light cylinder.

\subsubsection{Particle Energy Distribution}

As we have seen in the foregoing subsection, the distribution function of the particles forms a 'shock' in the Lorentz factor direction. In figure 4 , we present

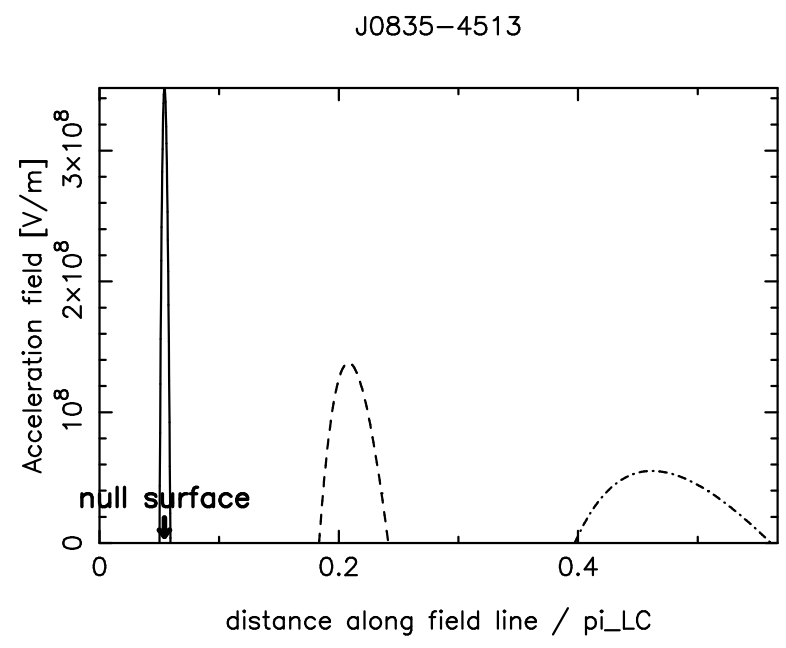

Fig. 2.- Spatial distribution of $E_{\|}(s)$ for $j^{\text {in }}=0$ (solid), 0.25 (dashed), and 0.5 (dash-dotted), for the Vela pulsar when $\alpha_{\mathrm{i}}=75^{\circ}$ and $j_{\text {gap }}=4.6 \times 10^{-5}$ and $j^{\text {out }}=0$. The abscissa designates $s / \varpi_{\mathrm{LC}}$, the distance along the last-open field line normalized by the light cylinder radius.

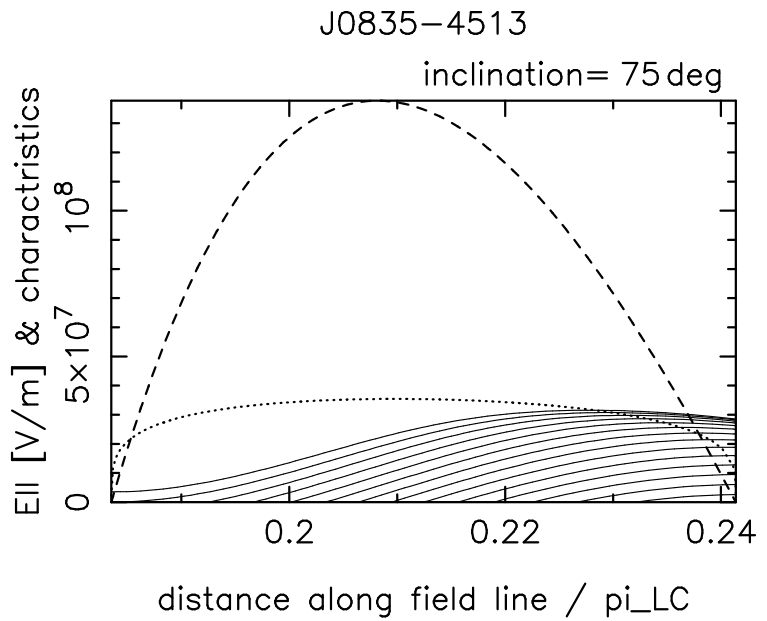

Fig. 3.- Spatial distribution of $E_{\|}(s)$ (dashed) for the Vela pulsar when $\alpha_{\mathrm{i}}=75^{\circ}, j^{\text {in }}=0.25, j^{\text {out }}=$ 0 , and $j_{\text {gap }}=4.6 \times 10^{-5}$. The characteristics for positrons are also shown by solid lines. The equilibrium Lorentz factor, which would be obtained if the curvature radiation-reaction force balanced the electrostatic acceleration, is indicated by the dotted line. 
the energy distribution of positrons at several representative points along the field line. At the inner boundary $\left(s=0.184 \varpi_{\mathrm{LC}}\right)$, particles are injected with Lorentz factors typically less than $4 \times 10^{6}$ as indicated by the solid line. Particles migrate along the characteristics in the phase space and gradually form a 'shock' as the dashed line (at $s=0.205 \varpi_{\mathrm{LC}}$ ) indicates, and attains maximum Lorentz factor at $s=0.228 \varpi_{\mathrm{LC}}$ as the dash-dotted line indicates. Then they begin to be decelerated gradually and escape from the gap with large Lorentz factors $\sim 2.8 \times 10^{7}$ (dotted line) at the outer boundary, $s=s^{\text {out }}=$ $0.241 \varpi_{\mathrm{LC}}$.

Even though the 'shock' is captured by only a few grid points for the dash-dotted line in figure 4, the CIP scheme accurately conserves the total current density,

$$
\begin{aligned}
j_{\text {tot }} & =\frac{2 \pi c e}{\Omega B(s)} \int_{1}^{\infty}\left[N_{+}(s, \Gamma)+N_{-}(s, \Gamma)\right] d \Gamma \\
& =j^{\text {in }}+j^{\text {out }}+j_{\text {gap }} \approx j^{\text {in }} .
\end{aligned}
$$

For this case, $j_{\text {tot }}$ is accurately conserved at 0.25 level within $0.2 \%$ errors even at the 'shock'.

\subsubsection{Formation of Power-law Gamma-ray Spec- trum}

So far, we have seen that the outwardly propagating particles are not saturated at the equilibrium value and that such particles escape from the gap with sufficient Lorentz factors suffering subsequent cooling via curvature process. It seems, therefore, reasonable to suppose that a significant fraction of the $\gamma$-ray luminosity is emitted from such escaping particles.

We present in figure 5 the $\gamma$-ray spectrum emitted from outwardly propagating particles (i.e., positrons) for the same case as in figure 3. The dashed line represents the $\gamma$-ray flux emitted within the gap, while the solid one includes that emitted outside of the gap by the escaping particles. Therefore, the difference between the solid and the dashed lines indicates the $\gamma$ ray flux emitted by the particles migrating outside of the gap. For comparison, we plot the phase-averaged EGRET spectrum, which is approximated by a power law with a photon index -1.7 (Kanbach et al. 1994) by open circles.

It follows from the figure that the $\gamma$-ray spectrum in $100 \mathrm{MeV}-3 \mathrm{GeV}$ energies can be explained by the curvature radiation emitted by the escaping particles. We adjusted the transfield thickness as

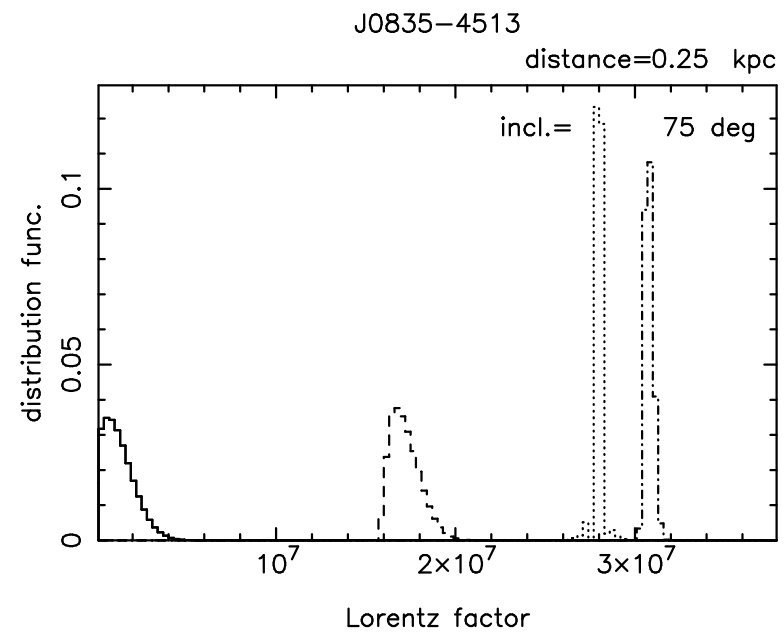

Fig. 4.- Particle energy distribution at several points along the magnetic field lines for the same case as in figure 3. Initial spectrum (solid line) evolves to dashed, dash-dotted, and dotted lines, as positrons propagate outwards.

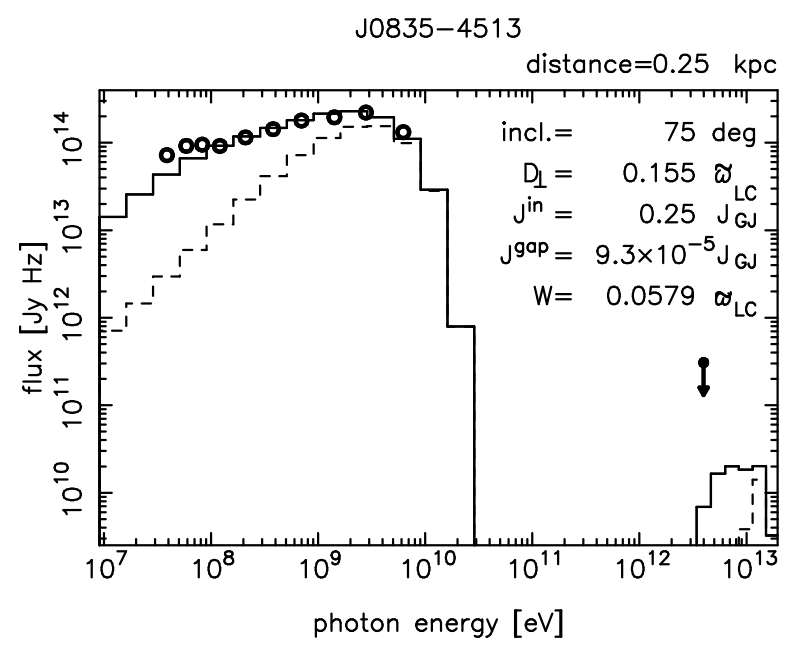

Fig. 5.- Computed $\gamma$-ray spectrum for the Vela pulsar for the same case as in figure 3. The dashed line depicts the flux emitted within the gap, while the solid one includes that emitted outside it. 
$D_{\perp}=0.16 \varpi_{\mathrm{LC}}=2.8 \mathrm{~W}$ so that the observed flux may be explained. The luminosity of the $\gamma$-rays emitted outside of the gap contribute $48 \%$ of the total luminosity $5.08 \times 10^{33} \mathrm{ergs} \mathrm{s}^{-1}$ between $100 \mathrm{MeV}$ and $20 \mathrm{GeV}$. In another word, we do not have to assume a power-law energy distribution for particles (as assumed in some of the previous outer-gap models) to explain the power-law $\gamma$-ray spectrum for the Vela pulsar. This conclusion is natural, because a power-law energy distribution of particles will not be achieved by an electrostatic acceleration, and because magnetohydrodynamic shocks (i.e., real shocks) will not be formed in the accelerator.

Because the X-ray field is dense for this young pulsar, the pair-production mean free path, and hence the gap width becomes small (for details, see Hirotani \& Okamoto 1998; Papers IV \& V). As a result, the potential drop in the gap $2.24 \times 10^{13} \mathrm{~V}$ is only $0.81 \%$ of the electro-motive force (EMF) exerted on the spinning neutron star surface $\sim \mu / \varpi_{\mathrm{LC}}^{2}=2.79 \times 10^{15} \mathrm{~V}$. Nevertheless, this potential drop is enough to accelerate particles into high Lorentz factors, $10^{7.5}$.

\subsubsection{Solutions in a Wide Parameter Space}

With the hydrogen atmosphere model, we can explain the observed $\gamma$-ray spectrum in a wide parameter space $45^{\circ}<\alpha_{\mathrm{i}}<75^{\circ}$ and $0.125<j^{\text {in }}<0.25$, by appropriately choosing $j_{\text {gap }}$ and $D_{\perp}$. With increasing $j_{\text {gap }}(\ll 1)$, the $\nu F_{\nu}[\mathrm{Jy} \mathrm{Hz}]$ peak energy increases because of the increased $W$, while the sub-GeV spectrum becomes hard because of the significant $\gamma$-ray emission within the extended gap (rather than outside of it). On the other hand, $D_{\perp}$ affects only the normalization of the $\gamma$-ray flux; the $\gamma$-ray luminosity is proportional to $D_{\perp}^{2}$.

Let us first fix $j^{\text {in }}$ at 0.25 and consider how the best-fit values of $j_{\text {gap }}$ and $D_{\perp}$ depend on $\alpha_{\mathrm{i}}$. As we have seen, they are $j_{\text {gap }}=4.6 \times 10^{-5}$ and $D_{\perp}=$ $0.155 \varpi_{\mathrm{LC}}=0.84 s^{\text {in }}$ for $\alpha_{\mathrm{i}}=75^{\circ}$. However, the ratio $D_{\perp} / s^{\text {in }}$ increases with decreasing $\alpha_{\mathrm{i}}$ and becomes 1.06 for $45^{\circ}$. From geometrical consideration, we conjecture that $D_{\perp}$ should not greatly exceed $s^{\text {in }}$; we thus consider that $\alpha_{\mathrm{i}}>45^{\circ}$ is appropriate for $j^{\text {in }}=0.25$. A large $\alpha_{\mathrm{i}}$ is preferable to obtain a small $D_{\perp} / \varpi_{\mathrm{LC}}$. However, since the radio pulsation shows a single peak, we consider that $\alpha_{\mathrm{i}}$ is not close to $90^{\circ}$. On these grounds, we adopted $\alpha_{\mathrm{i}}=75^{\circ}$ as a compromise for the Vela pulsar.

Let us next fix $\alpha_{\mathrm{i}}$ at $75^{\circ}$ and consider how the best- fit values of $j_{\text {gap }}$ and $D_{\perp}$ depend on $j^{\text {in }}$. The ratio $D_{\perp} / \varpi_{\mathrm{LC}}$ increases with decreasing $j^{\text {in }}$ and becomes 0.94 for $j^{\text {in }}=0.125$ (c.f. 0.84 for $j^{\text {in }}=0.25$ ). This is because the decreased flux of the outwardly migrating particles (due to decreased $j^{\text {in }}$ ) must be compensated by a large $D_{\perp}$ to produce the same $\gamma$-ray flux. Thus, we consider $j^{\text {in }}>0.125$ is appropriate for $\alpha_{\mathrm{i}}=75^{\circ}$. A large $j^{\text {in }}$ is preferable to obtain a small $D_{\perp} / \varpi_{\mathrm{LC}}$. However, for $j^{\text {in }}>0.25$, the gap is so extended that a significant $\gamma$-rays are emitted above $\mathrm{GeV}$ within the gap; as a result, the sub-GeV spectrum becomes too hard. On these grounds, we adopted $j^{\text {in }}=0.25$ as a compromize.

For $45^{\circ}<\alpha_{\mathrm{i}}<75^{\circ}, 0.125<j^{\text {in }}<0.25$, and appropriately chosen $j_{\text {gap }}$ and $D_{\perp}$, TeV flux is always less than $3 \times 10^{10} \mathrm{JyHz}$. Thus, one general point becomes clear: TeV flux is unobservable with current groundbased telescopes, provided that the emission solid angle is 1 ster and that the surface thermal (not magnetospheric) X-rays are upscattered inside and outside of the gap. Since the magnetospheric X-rays will be beamed away from the gap and their specific intensity is highly uncertain, we leave the problem of the upscatterings of magnetospheric (power-law) X-rays untouched.

\subsection{PSR B1706-44}

We next apply the theory to a Vela-type pulsar, PSR B1706-44. To consider $D_{\perp} / s^{\text {in }}$ as small as possible, we adopt a large magnetic inclination $75^{\circ}$. We compare $\nu F_{\nu}$ spectra for the three cases: $j^{\text {in }}=0.4$, 0.2 , and 0.1 .

To examine how the sub-GeV spectrum depends on $j^{\text {in }}$, we fix the $\nu F_{\nu}$ peak at the observed value, $\sim 2 \mathrm{GeV}$, by adjusting $j_{\text {gap }}$ appropriately. For the solid $\left(j^{\text {in }}=0.4\right)$, dashed $\left(j^{\text {in }}=0.2\right)$, and dashdotted $\left(j^{\text {in }}=0.1\right)$ lines in figure 6 , we adopt $j_{\text {gap }}=$ $2.2 \times 10^{-4}, 1.8 \times 10^{-4}$, and $1.5 \times 10^{-4}$, respectively, and $d=2.5 \mathrm{kpc}$. Moreover, the perpendicular thickness is adjusted so that the predicted flux may match the observed value $\left(3.2 \times 10^{13} \mathrm{JyHz}\right)$ at $1.4 \mathrm{GeV}$; for the solid, dashed, and dash-dotted lines, they are $D_{\perp}=1.05 \varpi_{\mathrm{LC}}=3.5 \mathrm{~s}^{\mathrm{in}}, 0.61 \varpi_{\mathrm{LC}}=4.1 \mathrm{~s}^{\mathrm{in}}$, $0.49 \varpi_{\mathrm{LC}}=5.3 \mathrm{~s}^{\mathrm{in}}$, respectively. The sub-GeV spectrum becomes hard with increasing $j^{\text {in }}$, because the ratio of the flux emitted outside of the gap and that emitted within it decreases as the gap extends with increasing $j^{\text {in }}$. However, as the solid line indicates, the obtained sub-GeV spectrum is still too soft to match the observation. For a smaller $j^{\text {in }}(<0.1)$, the 
sub-GeV spectrum becomes further soft. For a larger $j^{\text {in }}(>0.4), D_{\perp}$ exceeds $\varpi_{\text {LC }}$. For a non-zero $j^{\text {out }}$, the inwardly shifted gap is shrunk to emit smaller $\gamma$-ray flux, which results in a further greater $D_{\perp} / s^{\text {in }}$. On these grounds, the predicted sub-GeV spectrum becomes too soft or the $\gamma$-ray flux becomes too small (i.e., $D_{\perp}$ becomes too large) for any parameter set of $j^{\text {in }}$ and $j^{\text {out }}$, if $\alpha_{\mathrm{i}}=75^{\circ}$ and $d=2.5 \mathrm{kpc}$.

For a small inclination $\left(\alpha_{\mathrm{i}}<75^{\circ}\right)$, the gap is located relatively outside of the magnetosphere, because the null surface crosses the last-open field line at large distances from the star. Since $\lambda_{\mathrm{p}}$ increases in equation (29) at large distances from the star, the gap is extended for a small magnetic inclination. In such an extended gap, particles saturate at the equilibrium Lorentz factor in the outer part (Takata et al. 2002) and emit most of the $\gamma$-rays around the central energy of curvature radiation. As a result, a hard sub-GeV spectrum can be expected; however, we have to assume a large $D_{\perp}$ that exceeds $\varpi_{\mathrm{LC}}$ if $d=2.5 \mathrm{kpc}$.

Nevertheless, if $d$ is much less than $2.5 \mathrm{kpc}$ and $\alpha_{\mathrm{i}}<75^{\circ}$, we can explain the observed spectrum with moderate $D_{\perp}$. For example, for $d=1 \mathrm{kpc}, \alpha_{\mathrm{i}}=45^{\circ}$, $j^{\text {in }}=0.4$, and $j_{\text {gap }}=3.8 \times 10^{-3}$, the gap exists in $0.51 \varpi_{\mathrm{LC}}<s<0.74 \varpi_{\mathrm{LC}}$ and the resultant spectrum (dotted line in figure 6) matches the observation relatively well with a marginally acceptable thickness, $D_{\perp}=0.80 \varpi_{\mathrm{LC}}=1.5 \mathrm{~s}^{\mathrm{in}}$.

On the other hand, for a large inclination $\left(\alpha_{\mathrm{i}}>\right.$ $75^{\circ}$ ), the sub-GeV spectrum becomes softer than $\alpha_{\mathrm{i}}=$ $75^{\circ}$ case for the same $j^{\text {in }}, j^{\text {out }}$, and $j_{\text {gap }}$. Therefore, the spectrum will not match the observation whatever distance we may assume.

We can alternatively consider a CHR-like outer gap. Assuming a small $D_{\perp}$ (say, $0.05 \varpi_{\mathrm{LC}}$ ), we find that the gap extends along the field lines due to the screening effect of the zero-potential walls (i.e., the first term in the right-hand side of eq. [42]). In most portions of this extended gap, particles are nearly saturated at the equilibrium Lorentz factor. As a result, the sub-GeV spectrum becomes hard and match the observation with appropriate peak energy around $2 \mathrm{GeV}$. However, in this case, the $\gamma$-ray flux becomes too small to match the observed value, unless we adopt an unrealistic distance (300 pc for $D_{\perp}=0.05 \varpi_{\mathrm{LC}}$ ).

In short, the phase-averaged EGRET spectrum for this pulsar cannot be explained either by our current model or by the CHR picture $\left(D_{\perp} \ll W\right)$ for any combinations of $\alpha_{\mathrm{i}}, j^{\text {in }}, j^{\text {out }}, j_{\text {gap }}$, and $D_{\perp}$, if $d=$ $2.5 \mathrm{kpc}$. Therefore, we suggest a small distance (e.g., $1 \mathrm{kpc})$ for this pulsar with a large $D_{\perp}\left(\sim \varpi_{\mathrm{LC}}\right)$.

\subsection{The Geminga Pulsar}

Let us apply the theory to a cooling neutron star, the Geminga pulsar. For a small $\alpha_{\mathrm{i}}$ (e.g., $45^{\circ}$ ), the gap is so extended that the outer boundary exceeds the light cylinder. For a larger $\alpha_{\mathrm{i}}$, on the other hand, not only the outer-gap emission, but also a polar-cap one could be in our line of sight. Since there has been no radio pulsation confirmed, we consider a moderate magnetic inclination $\alpha_{\mathrm{i}}=60^{\circ}$.

Since the soft photon field is less dense compared with young pulsars like Vela or B1706-44, the gap is extended along the field lines. For the set of parameters $j^{\text {in }}=0.25, j^{\text {out }}=0$, and $j_{\text {gap }}=8.0 \times 10^{-5}$, which gives the best-fit $\gamma$-ray spectrum, we obtain $W=0.59 \varpi_{\mathrm{LC}}$. We present the spatial distribution of $E_{\|}$obtained for this set of parameters as the dashed line in figure 7 , as well as the characteristics (solid lines). In the outer part of this extended gap, $\rho_{\mathrm{GJ}}$, which gradually increases, is partially canceled by the $-\Psi / D_{\perp}^{2}$ term in equation (42). As a result, $E_{\|}(s)$ deviates from quadratic distribution and decrease gradually as well. Because of this extended structure, particles are nearly saturated at the equilibrium Lorentz factor (dotted line in fig. 7). In another word, the mono-energetic approximation adopted in Papers I-IX is justified for this middleaged pulsar. Particle distribution function forms a strong 'shock', which is captured only with one grid point, in $0.4<s / \varpi_{\mathrm{LC}}<0.45$. As a result, $j_{\text {tot }}$ fluctuates a little as figure 8 indicates. Nevertheless, it returns to the 0.2525 level, which is $1 \%$ greater than the value it should be (0.250), as the characteristics begin to be less concentrated beyond $s=0.45 \varpi_{\mathrm{LC}}$.

In figure 9 , we present the resultant $\gamma$-ray spectrum. Because of the nearly saturated motion of the particles, they lose most of their energy within the gap. As a result, $\gamma$-ray luminosity associated with the escaping particles $\left(5.3 \times 10^{31} \mathrm{ergs} \mathrm{s}^{-1}\right)$, is negligibly small compare to that emitted within the gap $\left(1.11 \times 10^{33} \mathrm{ergs} \mathrm{s}^{-1}\right)$, which is represented by the dashed line.

It should be noted that a small $D_{\perp}=0.17 \varpi_{\mathrm{LC}}=$ $0.28 \mathrm{~W}$ gives the best-fit spectrum. This perpendicularly thin gap indicates that the solution presented in fig. 7, 8, and 9 are, in fact, obtained in the CHR pic- 


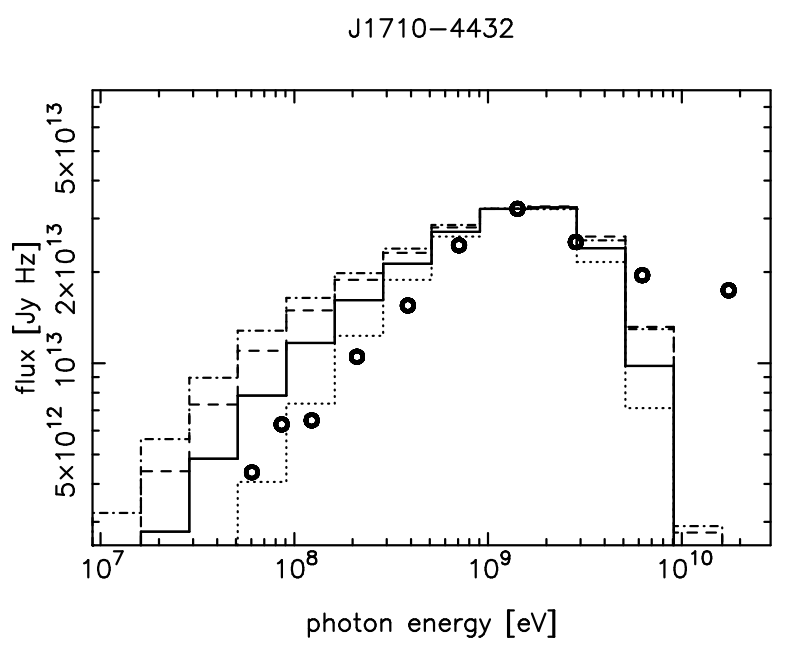

Fig. 6.- Computed $\gamma$-ray spectra for PSR B170644. The solid, dashed, dash-dotted lines represent the spectra for $j^{\text {in }}=0.4,0.2,0.1$, respectively, when $d=2.5 \mathrm{kpc}$ and $\alpha_{\mathrm{i}}=75^{\circ}$, while the dotted one for $j^{\text {in }}=0.4$ when $d=1.0 \mathrm{kpc}$ and $\alpha_{\mathrm{i}}=45^{\circ}$.

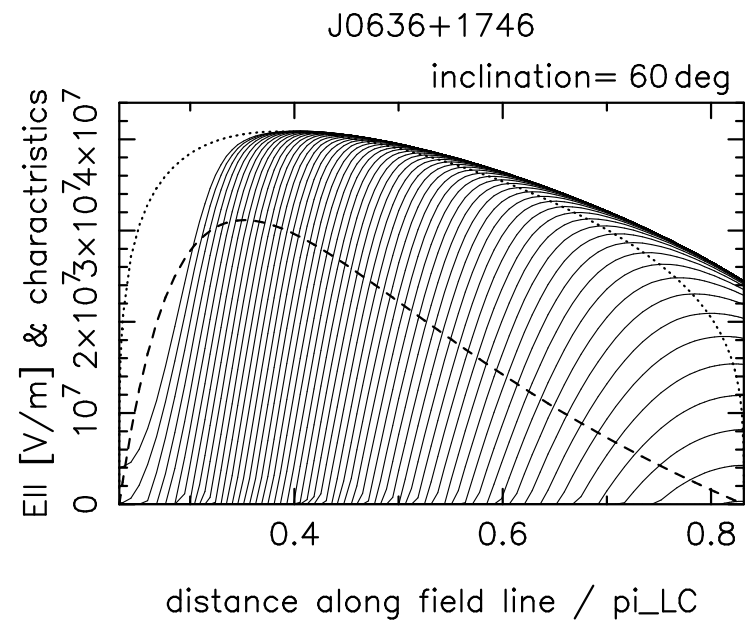

Fig. 7.- Spatial distribution of $E_{\|}(s)$ (dashed line) for the Geminga pulsar when $\alpha_{\mathrm{i}}=60^{\circ}, j^{\text {in }}=0.25$, $j^{\text {out }}=0$, and $j_{\text {gap }}=8.0 \times 10^{-5}$. Particles are saturated at the equilibrium Lorentz factor (dotted line) for this middle-aged pulsar.

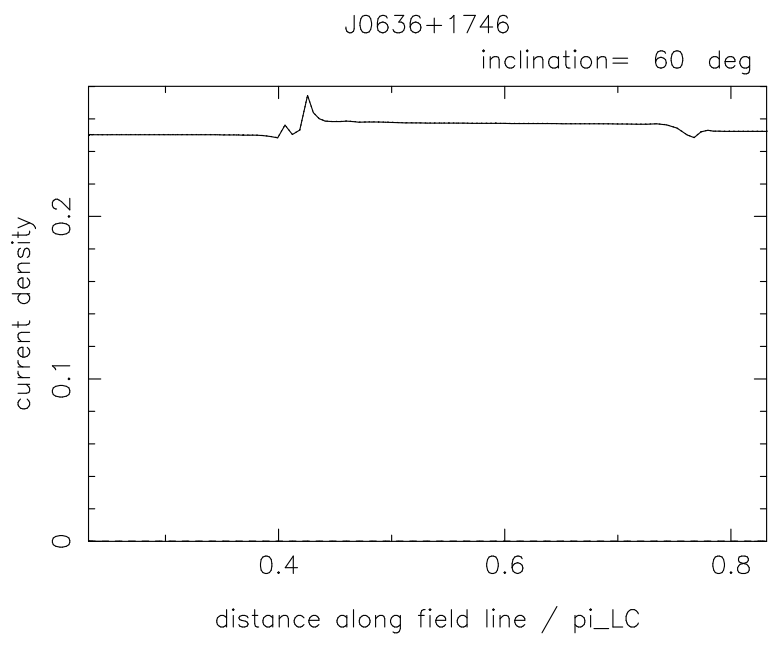

Fig. 8. - Total current density, $j_{\text {tot }}$, for the same case as in figure 7 . Even though the particle distribution function forms a strong 'shock' in the Lorentz factor direction in $0.4 \varpi_{\mathrm{LC}}<s<0.45 \varpi_{\mathrm{LC}}$ (see fig. 7 ), $j^{\text {tot }}$ is conserved relatively accurately.

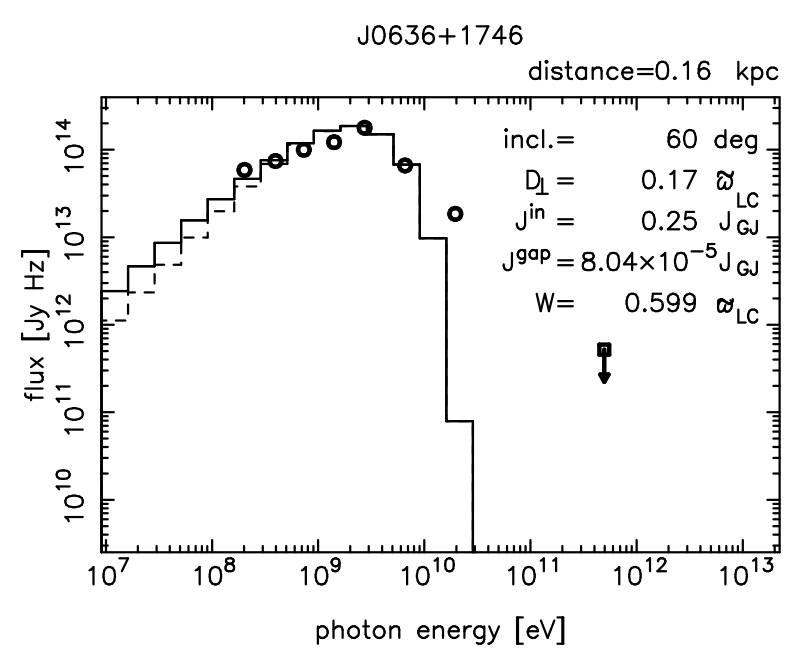

Fig. 9.- Computed $\gamma$-ray spectrum for the Geminga pulsar for the same case as in figure 7 . The dashed and solid lines represent the same components as figure 5 . 
ture $\left(D_{\perp} \ll W\right)$ in the sense that the screening effect due to the zero-potential walls (i.e., $-\Psi / D_{\perp}^{2}$ term in eq. [42]) is important. There are, of course, differences from previous works: In the present work, the set of Maxwell and Boltzmann equations are solved, while the two-dimensional screening effects are considered only approximately. To consider the twodimensional effect more rigorously, we have to solve the elliptic-type partial differential equation (4) on the poloidal plane, together with ordinary differential equations (53) and hyperbolic-type partial differential equations (44) and (45) simultaneously.

It follows from figure 9 that we can explain the observed spectrum between $200 \mathrm{MeV}$ and $6 \mathrm{GeV}$ by superposing the spectra of $\gamma$-rays emitted at various point. It is interesting to compare this result with what obtained for the Vela pulsar (fig. 5). Between $100 \mathrm{MeV}$ and $1 \mathrm{GeV}$ energies, both spectra are formed by the superposition of the curvature radiation emitted by the particles having different energies at different positions. The important difference is that the particles are saturated at the equilibrium Lorentz factor in the gap for the Geminga pulsar, while they are nearly mono-energetic but only decelerated via curvature process outside of the gap for the Vela pulsar. Because the particles are no longer accelerated outside of the gap, they emit $\gamma$-rays in lower energies compared with those still being accelerated in the gap. As a result, the $\gamma$-ray spectrum for the Vela pulsar becomes softer than that for the Geminga pulsar. Extending this consideration, we can predict that a $\gamma$-ray spectrum below $\mathrm{GeV}$ is soft for a young pulsar and tends to become hard as the pulsar ages.

\subsection{PSR B1055-52}

Let us finally apply the present theory to another middle-aged pulsar, B1055-52. To obtain a large $\gamma$ ray flux for an appropriately chosen set of $j^{\text {in }}, j_{\text {gap }}$, and $D_{\perp}\left(<s^{\text {in }}\right)$, we adopt a large magnetic inclination, $\alpha_{\mathrm{i}}=75^{\circ}$.

Since the acceleration field and the particle energy distributions are similar to the Geminga pulsar, we present only the computed $\gamma$-ray spectra for this pulsar in figure 10. The solid and dashed lines represent the spectra for the $j^{\text {in }}=0.25$ and 0.125 , respectively. For $j^{\text {in }}=0.25, j_{\text {gap }}=2.9 \times 10^{-3}$ and $D_{\perp}=0.27 \varpi_{\mathrm{LC}}=0.98 \mathrm{~W}$ are chosen so that the peak energy of curvature radiation may match the observed peak energy. In this case, the gap exists in $0.1485 \varpi_{\mathrm{LC}}<s<0.4120 \varpi_{\mathrm{LC}}$. For $j^{\text {in }}=0.125$, $j_{\text {gap }}=3.7 \times 10^{-3}$ and $D_{\perp}=0.20 \varpi_{\mathrm{LC}}=1.7 \mathrm{~W}$ are chosen and the gap exists in $0.0870 \varpi_{\mathrm{LC}}<s<0.201 \varpi_{\mathrm{LC}}$. It is interesting to note that the spectrum softens for a smaller $D_{\perp} / W$. This is because the zero-potential wall partially screens $E_{\|}$in equation (42). For the middle-aged pulsars Geminga and B1055-52, the $\gamma$ ray luminosity is not simply proportional to $D_{\perp}^{2}$, because of this screening effect.

It follows from the figure that the solid line matches the observed flux with an unreasonable transfield thickness, $D_{\perp}=1.8 \mathrm{~s}^{\mathrm{in}}$. For the dashed line, we have to choose $D_{\perp}=2.3 \mathrm{~s}^{\mathrm{in}}$. The observed fluxes cannot be explained with acceptable gap width (e.g., $D_{\perp}<s^{\text {in }}$ ) no matter what we may adjust $j^{\text {in }}, j^{\text {out }}, j_{\text {gap }}$, and $D_{\perp}$ if $d=1.53 \mathrm{kpc}$.

On these grounds, we conjecture that the distance $1.5 \pm 0.4 \mathrm{kpc}$ determined from the dispersion measure (Taylor \& Cordes 1993) is too large and that a more closer distance, such as $500 \mathrm{pc}$ derived from ROSAT data analysis (Ögelman \& Finley 1993) or 700 pc estimated from a study of the extended nonthermal radio source around the pulsar (Combi, Romero, Azcárate 1997), is plausible. For example, if we set $d=1 \mathrm{kpc}$, we can fit the spectrum with a reasonable transverse thickness, $D_{\perp}=1.06 \mathrm{~s}^{\mathrm{in}}=0.15 \varpi_{\mathrm{LC}}=0.45 \mathrm{~W}$, for $j^{\text {in }}=0.25$ and $j_{\text {gap }}=4.5 \times 10^{-3}$ (dotted line in fig. 10). For such a transversely thin $\left(D_{\perp}=0.45 \mathrm{~W}\right)$ gap, $E_{\|}$is significantly screened by the zero-potential wall to become CHR-like distribution, as in the case for the Geminga pulsar.

In Papers IX and X, we suggested a smaller distance, $d<0.5 \mathrm{kpc}$, using ROSAT and ASCA data (Greiveldinger et al. 1996), which gives an enormously large blackbody area $A_{\mathrm{s}}=7.3 A_{*}(d / 1.53 \mathrm{kpc})^{2}$. However, a more recent BeppoSAX data suggest a reasonable blackbody area of $A_{\mathrm{s}}=1.3 A_{*}$; as a result, a larger distance $d<1 \mathrm{kpc}$ becomes acceptable in the present analysis.

\section{Discussion}

In summary, we have quantitatively examined the stationary pair-production cascade in an outer magnetosphere, by solving the set of Maxwell and Boltzmann equations one-dimensionally along the magnetic field lines. We revealed that an accelerator (or a potential gap) is quenched by the created pairs in the gap but is not quenched by the injected particles from outside of the gap, and that the gap position shifts as a function of the injected particle fluxes: If 
the injection rate across the inner (or outer) boundary approaches the typical Goldreich-Julian value, the gap is located near to the light cylinder (or the star surface). It should be emphasized that the particle energy distribution is not represented by a power law, as assumed in some of previous outer-gap models. The particles escape from the gap with sufficient Lorentz factors and emit significant photons in $100 \mathrm{MeV}$ $3 \mathrm{GeV}$ energies via curvature radiation outside of the gap. The $\gamma$-ray spectrum including this component explains the phase-averaged EGRET spectra for the Vela pulsar (and also for PSR B1706-44 with a small distance, $d<1 \mathrm{kpc}$ ). As a pulsar ages, its outer gap extends in the magnetosphere to approach the CHR solution: For the two middle-aged pulsars Geminga and B1055-52, we obtain harder spectra compared with the two young pulsars. TeV fluxes are unobservable with current ground-based telescopes for all the four pulsars.

We consider the stability of such a gap in the next subsection. We then point out an implication to the $\gamma$-ray luminosity versus the spin-down luminosity in $\S 6.2$, and discuss future extensions of the present method in $\S \S 6.3-6.5$.

\subsection{Stability of the Gap}

Let us discuss the electrodynamic stability of the gap, by considering whether an initial perturbation of the gap width $W$ grows or not. Imagine that $W$ increases perturbatively. Then the Maxwell equation (4) increases $\left|E_{\|}\right| \equiv-\partial \Psi / \partial s$. To see this behavior more clearly, we can Taylor expand the right-hand side around the point $s=s_{0}$ where $\rho_{\mathrm{e}}-\rho_{\mathrm{GJ}}$ vanishes. Neglecting $z$ dependence, we obtain

$$
-\frac{d^{2} \Psi}{d s^{2}}=-4 \pi A\left(s-s_{0}\right) ;
$$

the constant $A$ is of the order of $\Omega \mu_{\mathrm{m}} /\left(c \varpi_{\mathrm{LC}}^{4}\right)$, where $\mu_{\mathrm{m}}$ is the magnetic dipole moment of the star (for an explicit expression of $A$, see eq. [3] in Paper V). If $W \ll \varpi_{\mathrm{LC}}, s_{0}$ approaches $s_{\text {cnt }}$ (eq. [37]). Integrating equation (64), we obtain $E_{\|}(s)=2 \pi A\left(s^{\text {out }}-s\right)(s-$ $\left.s^{\text {in }}\right)$, where $s^{\text {out }}=s^{\text {in }}+W$. Thus, the averaged $E_{\|}$in the gap can be evaluated as

$$
\left\langle E_{\|}\right\rangle=\frac{1}{W} \int_{s^{\text {in }}}^{s^{\text {out }}} E_{\|}(s) d s=\frac{\pi}{3} A W^{2}
$$

Particle Lorentz factor $\Gamma$ also increases with increasing $W$. For example, if particles are unsaturated, particles energy is roughly proportional to the potential drop; thus, we obtain

$$
\Gamma \sim\left\langle E_{\|}\right\rangle \cdot W \propto W^{3} .
$$

On the contrary, if they are saturated, the curvature radiation drag gives

$$
\Gamma \sim\left(\frac{3 \rho_{\mathrm{c}}\left\langle E_{\|}\right\rangle}{2 e}\right)^{1 / 4} \propto W^{1 / 2}
$$

In general, $\Gamma$ depends on $W^{\alpha}$ with $0.5<\alpha<3$; thus, $\Gamma$ increases with increasing $W$ irrespectively whether the particles are saturated or not.

As a result of this increased $\Gamma$, the $\gamma$-ray energy, $h \nu_{\gamma}$, increases. This is because the central energy of the curvature radiation increases with increasing $\Gamma$ (eq. [23]). Moreover, the number of $\gamma$-rays emitted by a single particle, $N_{\gamma} \propto W \cdot \Gamma$, increases with increasing $W$ (eq. [22] \& [28]). The flowchart is depicted in figure 11.

The increased $h \nu_{\gamma}$ results in a decrease of $E_{\text {th }}$ by equation (13). The decreased $E_{\text {th }}$ leads to the increase of $\eta_{\mathrm{p}}$ by equation (12), and hence to the decrease of $\lambda_{\mathrm{p}}$ by equation (17). This result does not depend on the origin of the X-ray field. For example, if the Xrays are thermal origin, $\lambda_{\mathrm{p}}$ decreases with decreasing threshold energy $E_{\mathrm{th}}$, because the specific intensity of the X-ray field will be unchanged. If the X-rays are magnetospheric origin, on the other hand, $\lambda_{\mathrm{p}}$ decreases more sharply than the thermal case, because the X-ray density illuminating the gap will increase with increasing pair-production rate outside of the gap due to the increased $h \nu_{\gamma}\left(\right.$ and $N_{\gamma}$ ).

Finally, both the decreased $\lambda_{\mathrm{p}}$ and the increased $N_{\gamma}$ contribute to reduce the initial increase of $W$ by equation (29). Because of this negative feedback effect, stationary solutions exist in a wide range of pulsar parameters (from young to middle-aged pulsars). On these grounds, although the perturbation equations are not solved under appropriate boundary conditions for the perturbed quantities, we conjecture that an outer gap is electrodynamically stable, irrespective whether the X-ray field illuminating the gap is thermal or non-thermal origin.

\subsection{Gamma-ray vs. Spin-down Luminosities}

It should be noted that the emission from the escaping particles attain typically $40 \%$ of the total $\gamma$ ray luminosity for young pulsars. Thus, it is worth 
mentioning its relationship with the spin-down luminosity,

$$
L_{\text {spin }}=-I \Omega \dot{\Omega} \propto \Omega^{n+1},
$$

where the braking index $n$ is related to the spin-down rate as

$$
\dot{\Omega}=-k \Omega^{n} .
$$

If the spin down is due to the magnetic dipole radiation, we obtain $n=3$.

The outwardly propagating particles escape from the gap with spatial number density

$$
N^{\text {out }}=\left(j^{\text {in }}+j_{\text {gap }}\right) \frac{\Omega B^{\text {out }}}{2 \pi c e},
$$

where $B^{\text {out }}=B\left(s^{\text {out }}\right)$. Therefore, the energy carried by the escaping particles per unit time is given by

$$
L_{\mathrm{esc}}=D_{\perp}^{2} c N^{\mathrm{out}} \Gamma_{\mathrm{esc}} m_{\mathrm{e}} c^{2},
$$

where $\Gamma_{\text {esc }}\left(\sim 10^{7.5}\right)$ refers to the Lorentz factor of escaping particles. Note that $\Gamma_{\text {esc }}$ is essentially determined by the equilibrium Lorentz factor (dotted line in fig. 3) near the gap center. Since the equilibrium Lorentz factor depends on the one-fourth power of $E_{\|}$, the variation of $\Gamma_{\text {esc }}$ on pulsar parameters is small. We can approximate $B^{\text {out }}$ as

$$
B^{\text {out }} \sim \frac{\mu_{\mathrm{m}}}{\varpi_{\mathrm{LC}}^{3}}\left(\frac{\varpi_{\mathrm{LC}}}{r^{\text {out }}}\right)^{3},
$$

where $r^{\text {out }}$ refers to the distance of the outer boundary of the gap from the star center. Let us assume that the position of the gap with respect to the light cylinder radius, $r^{\text {out }} / \varpi_{\mathrm{LC}}$, does not change as the pulsar evolves; this situation can be realized if $j^{\text {in }}-j^{\text {out }}$ is unchanged. Evaluating $B$ at $r=0.5 \varpi_{\mathrm{LC}}$, we obtain

$$
\begin{aligned}
L_{\mathrm{esc}} & =\frac{4 \Gamma_{\mathrm{esc}} m_{\mathrm{e}} c}{\pi e} \mu_{\mathrm{m}} \Omega^{2}\left(\frac{D_{\perp}}{\varpi_{\mathrm{LC}}}\right)^{2} \\
& \propto L_{\mathrm{spin}}{ }^{0.5}
\end{aligned}
$$

where $n=3$ is assumed in the second line. To derive this conclusion, it is essential that the particles are not saturated at the equilibrium Lorentz factor. Thus, the same discussion can be applied irrespective of the gap position or the detailed physical processes involved. For example, an analogous conclusion was derived for a polar-cap model by Harding, Muslimov, and Zhang (2002). It is, therefore, concluded that the observed relationship $L_{\gamma} \propto L_{\text {spin }}{ }^{0.5}$ merely reflects the fact that the particles are unsaturated in the gap and does not discriminate the gap position.
Let us compare this result with what would be expected in the CHR picture. Since the gap is extended significantly along the field lines in the CHR picture, particles are saturated at the equilibrium Lorentz factor to lose most of their energies within the gap, rather than after escaping from it. We can therefore estimate the $\gamma$-ray luminosity as

$$
L_{\text {gap }}=\left(D_{\perp} D_{\phi} W\right) \cdot N^{\text {out }} \cdot P_{\mathrm{CV}}
$$

where $D_{\phi}$ refers to the azimuthal thickness of the gap. Noting that the particle motion saturates at the equilibrium Lorentz factor satisfying $P_{\mathrm{CV}} / c=e E_{\|}$, recalling that the acceleration field is given by $E_{\|} \approx$ $\Omega B D_{\perp}^{2} / 4 \rho_{\mathrm{c}} c$ in the CHR picture, and evaluating $B$ at $r=\varpi_{\mathrm{LC}}$, we obtain

$$
\begin{aligned}
L_{\text {gap }} & =\frac{\mu_{\mathrm{m}}^{2} \Omega^{4}}{4 \pi c^{3}} \frac{D_{\perp}^{3} D_{\phi} W}{\varpi_{\mathrm{LC}}^{5}}\left(\frac{\rho_{\mathrm{c}}}{0.5 \varpi_{\mathrm{LC}}}\right)^{-1} \\
& \propto L_{\text {spin }},
\end{aligned}
$$

where $n=3$ is assumed again in the second line. Even though the escaping particles little contribute to the $\gamma$-ray luminosity in the CHR picture, it is worth mentioning the work done by Crusius-Wätzel and Lesch (2002), who accurately pointed out the importance of the escaping particles in the CHR picture, when we interpret $L_{\gamma} \propto L_{\text {spin }}^{0.5}$ relation.

As we have seen, the particles being no longer accelerated contribute for the $\gamma$-ray luminosity that is proportional to $L_{\mathrm{spin}}^{0.5}$. Reminding that the particles migrate with larger Lorentz factors than the equilibrium value in the outer part of the gap (see fig. 3), we can expect roughly half of the $\gamma$-ray luminosity is proportional to $L_{\text {spin }}^{0.5}$ (mainly between $100 \mathrm{MeV}$ and $1 \mathrm{GeV}$ ), and the rest of the half to $L_{\text {spin }}$ (mainly above $1 \mathrm{GeV}$ ). As a pulsar ages, its declined surface emission results in a large pair-production mean free path, and hence $W$. Because $\left|\rho_{\mathrm{GJ}}\right| \propto r^{-3}$ becomes small in the outer part of such an extended gap, $E_{\|}(s)$ deviates from quadratic distribution to decline gradually in the outer part (fig. 7). As a result, particles tend to be saturated at the equilibrium value. On these grounds, we can predict that the $\gamma$-ray luminosity tends to be proportional to $L_{\text {spin }}$ with age, deviating from $L_{\text {spin }}^{0.5}$ dependence for young pulsars.

In the present paper, we have examined the set of Maxwell and Boltzmann equations one-dimensionally both in the configuration and the momentum spaces (i.e., only $s$ and $\Gamma$ dependences are considered.) In the next three sections, we discuss the extension of the present method into higher dimensions 


\subsection{Returning Particles}

If we consider the pitch-angle dependence of particle distribution functions, we can compute the radiation spectrum with synchro-curvature formula (Cheng and Zhang 1996). Moreover, we can also consider the returning motion of particles inside and outside of the gap. The returning motion becomes particularly important when both signs of charge are injected across the boundary. For example, not only positrons but also electrons could be injected across the inner boundary from the polar-cap accelerator. If $E_{\|}>0$ for instance, the injected electrons return in the gap. This returning motion significantly affects the Poisson equation, if their injection rate is a good fraction of the Goldreich-Julian value.

It remains an unsettled issue whether an outer-gap accelerator resides on the field lines on which a polarcap accelerator exists. To begin with, let us consider the case when the plasma flowing between the polar cap and the outer-gap accelerator is completely charge separated. Such a situation can be realized, for instance, if only positively charged particles are ejected outwardly from the polar cap while there is virtually no electrons ejected inwardly from the outer gap. Neglecting the pair production, current conservation law gives the charge density, $\rho_{\mathrm{e}}$, per unit magnetic flux tube as

$$
\frac{\rho_{\mathrm{e}}}{B} \propto \frac{j_{\text {tot }}}{v},
$$

where $v$ refers to the particle velocity along the field line, and $j_{\text {tot }}$ the conserved current density per magnetic flux tube. At each point along the field line, $\rho_{\mathrm{e}}$ should match $\rho_{\mathrm{GJ}}$. If the field line intersects the null surface, $\rho_{\mathrm{e}}$ must vanish there; this obviously violates the causality in special relativity. Therefore, a stationary ejection of a completely charge-separated plasma from the polar cap can be realized only along the field lines between the magnetic axis and those intersecting the null surface at the light cylinder. On these grounds, it was argued that an outer-gap accelerator, which is formed close to the last-open field line, may not resides on the same field lines on which a polar-cap accelerator resides. This has been, in fact, the basic idea that an outer gap will not be quenched, because the particles ejected from the polar cap will flow along the different field lines. This idea was welcomed in outer-gap models, because a gap has been considered to be quenched if the external particle injection rate becomes comparable to the Goldreich-
Julian value, which was proved to be incorrect in this paper.

In general, however, the plasmas are not completely charge separated and consist of both signs of charge (e.g., positrons and electrons). Such a situation can be realized, for instance, if both charges are ejected outwardly from a polar-cap accelerator, or if positively charged particles are ejected outwardly from the polar cap while electrons are ejected inwardly from the outer gap, or if there is a pair production between the two accelerators. In these cases, the velocities of both charges will be adjusted so that both the current conservation and $\rho_{\mathrm{e}}=\rho_{\mathrm{GJ}}$ are satisfied at each point along the field lines. Therefore, it seems likely that a polar-cap accelerator and an outer-gap accelerator reside on the same field lines.

To examine if there is a stationary plasma flow between the polar cap and the outer gap, we must extend the present analysis into two dimensional momentum space in the sense that the pitch-angle dependence of the particle distribution functions is taken into account in addition to the Lorentz factor dependence. For example, if both charges are ejected from the polar-cap accelerator, electrons will return in the outer gap, screening the original acceleration field in the gap, and violating the original balance of $\rho_{\mathrm{e}}=\rho_{\mathrm{GJ}}$ outside of the gap. Because the returning motion of particles can be treated correctly if we consider the pitch-angle evolution of the distribution functions, and because the pair production is already taken into account, our present method is ideally suited to investigate the plasma flows and $E_{\|}$ distribution self-consistently inside and outside of the gap.

\subsection{Unification of Outer-gap Models}

In addition to the extension into a higher dimensional momentum space, it is also important to extend the present method into a two- or three-dimensional configuration space. In particular, determination of the perpendicular thickness, $D_{\perp}$, is important to constrain gap activities. There have been, in fact, some attempts to constrain $D_{\perp}$ in the CHR picture. Since $E_{\|}$is proportional to $B D_{\perp}^{2}$ if $D_{\perp} \ll W$, particles energies, and hence the $\gamma$-ray energies increase with increasing $D_{\perp}$ (for a fixed $B$ ). Zhang and Cheng (1997) constrained $D_{\perp}$, by considering the condition that the $\gamma$-rays cause photon-photon pair production in the gap. Subsequently, Cheng, Ruderman, and Zhang (2000) extended this idea into three-dimensional mag- 
netosphere and discussed phase-resolved $\gamma$-ray spectra for the Crab pulsar. In addition, Romani (1996) discussed the evolution of the $\gamma$-ray emission efficiency and computed the phase-resolved spectra for the Vela pulsar, by assuming that $B D_{\perp}^{2}$ declines as $r^{-1}$. However, in these works, screening effects due to pair production has not been considered; thus, the obtained $D_{\perp}$, as well as the hypothesized gap position along the magnetic fields, are still uncertain.

On the other hand, in our approach (picture), $D_{\perp}$ is not solved but only adjusted so that the $\gamma$-ray flux may match the observations. Therefore, the question we must consider next is to solve such geometrical and electrodynamical discrepancies between these two pictures. We can investigate this issue by extending the present method into higher spatial dimensions.

Furthermore, close to the last-open field line, $\rho_{\mathrm{e}}$ may fail to match $\rho_{\mathrm{GJ}}$ outside of the gap, because the emitted $\gamma$-rays soon propagate away from it and the pair production is expected to be less efficient. In such a highly charge-starved region in the magnetosphere, the electromagnetic field may be approximated by the Deutsch field (Deutsch 1955). Therefore, the $\gamma$-rays emitted close to the last-open field line may show a very hard spectrum as Higgins and Henriksen $(1997 ; 1998)$ predicted. To consider this issue further, we have to solve the set of Maxwell and Boltzmann equations on the poloidal plane $(s, z)$ both inside and outside of the gap, taking account of the deviation of the $\gamma$-rays from the field lines on which they were originally emitted.

\subsection{Unification of Outer-gap and Polar-cap Models}

Electrodynamically speaking, the essential difference between outer-gap and polar-cap accelerators is the value of the optical depth for pair production. In an outer-gap accelerator, pair production takes place via $\gamma-\gamma$ collisions and its mean-free path is much greater than the light cylinder radius. Therefore, a pair production cascade takes place gradually in the gap. In such a gap, $E_{\|}$is automatically screened out at both the boundaries by the Goldreich-Julian charge density, which varies monotonically along the field lines. For example, if $E_{\|}$is positive, $B_{\zeta}$ in equation (42) increases outwards: $d E_{\|} / d s>0$ in the inner part of the gap, while $d E_{\|} / d s<0$ in the outer part. Thus, we do not have to contrive a mechanism to screen out $E_{\|}$at the boundaries.
On the contrary, in a polar-cap accelerator, $E_{\|}$ cannot be screened out by $\rho_{\mathrm{GJ}}$. Nevertheless, in the vicinity of the star, the strong magnetic field (e.g., $B \sim 10^{12} \mathrm{G}$ ) leads to a magnetic pair production, of which mean free path is much less than the star radius. As a result, a pair production avalanche takes place in a limited region, which is called as the 'pair formation front', in the gap (Fawley, Arons, \& Sharlemann 1977; Harding \& Muslimov 1998, 2001, 2002; Shibata, Miyazaki, Takahara 1998, 2002; Harding, Muslimov, Zhang 2002). In the pair formation front, a small portion of the particles return to screen out $E_{\|}$. Such a returning motion can be self-consistently solved together with $E_{\|}$by our present method, if we implement the magnetic pair production and the resonant IC scattering redistribution functions in the source terms of the particles' and $\gamma$-rays' Boltzmann equations. We can execute the same advection-phase computation in CIP scheme; thus, all we have to do is to add these source terms in the non-advectionphase computation, which is not very difficult. Since analogous boundary conditions (e.g., $E_{\|}=0$ for a space-charge limited flow) will be applied, we expect the present method is also applicable to a polar-cap accelerator. This is an issue to be examined in our subsequent papers.

The author wishes to express his gratitude to Drs. A. K. Harding, S. Shibata, and K. S. Cheng for fruitful discussion on theoretical aspects, and to Drs. K. Shibata and A. Figueroa-Vinās for valuable advice on numerical analysis.

\section{REFERENCES}

Akhiezer, A. I., Berestetskii, V. B. 1965, in Quantum Electrodynamics (Wiley; New York), p. 450

Becker, W., Trümper, J. 1996, A\&AS 120, 69

Bekenstein, J. D., Oron, E. 1978, Phys. Rev. D. 18, 1809

Beskin, V. S., Istomin, Ya. N., Par'ev, V. I. 1992, Sov. Astron. 36(6), 642

Caraveo, P. A., Bignami, G. F., Mignani, R., Taff, L. G. 1996, ApJ 461, L91

Cha, A. N., Sembach, K. R., Danks, A. C. 1999, ApJ $515, \mathrm{~L} 25$

Cheng, K. S., Ho, C., Ruderman, M., 1986a ApJ, 300, 500 
Cheng, K. S., Ho, C., Ruderman, M., 1986b ApJ, 300, 522

Cheng, K. S., Zhang, L. 1996, ApJ 463, 271

Cheng, K. S., Ruderman, M., Zhang, L. 2000, ApJ, 537, 964

Chiang, J., Romani, R. W. 1992, ApJ, 400, 629

Chiang, J., Romani, R. W. 1994, ApJ, 436, 754

Combi, J. A., Romero, G. E., Azcárate 1997, ApSS, 250,1

Crusius-Wätzel, A. R., Lesch, H. 2002, submitted to Astroparticle Phys.

Daugherty, J. K., Harding, A. K. 1982, ApJ, 252, 337

Daugherty, J. K., Harding, A. K. 1996, ApJ, 458, 278

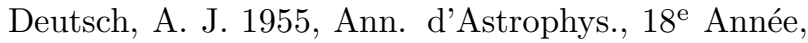
No. 1,1

Dermer, C. D., Sturner, S. J. 1994, ApJ, 420, L75

Fawley, W. M., Arons, J., Scharlemann, E. T. 1977, ApJ 217, 227

Fierro, J. M., Michelson, P. F., Nolan, P. L., Thompson, D. J., 1998, ApJ 494, 734

Goldreich, P. Julian, W. H. 1969, ApJ. 157, 869

Gotthelf, E. V., Halpern, J. P., Dodson, R. 2002, ApJ $567, \mathrm{~L} 125$

Greiveldinger, C., Camerini, U., Fry, W., Markwardt, C. B., Ögelman, H., Safi-Harb, S., Finley, J. P., Tsuruta, S. 1996, ApJ 465, L35

Halpern, J. P. Wang, Y. H., 1997, ApJ 477, 905

Harding, A. K., Tademaru, E., Esposito, L. S. 1978, ApJ, 225, 226

Harding, A. K., Muslimov, A. G., 1998, ApJ, 508, 328

Harding, A. K., Muslimov, A. G., 2001, ApJ, 556, 987

Harding, A. K., Muslimov, A. G., 2002, ApJ, 568, 862

Harding, A. K., Muslimov, A. G., B. Zhang, 2002, ApJ, 576, 366

Higgins, M. G., Henriksen R. N. 1997, MNRAS 292, 934

Higgins, M. G., Henriksen R. N. 1998, MNRAS 295, 188

Hirotani, K. 2000a, MNRAS 317, 225 (Paper IV)

Hirotani, K. 2000b, PASJ 52, 645 (Paper VI)

Hirotani, K. 2001, ApJ 549, 495 (Paper V)

Hirotani, K. Okamoto, I., 1998, ApJ, 497, 563
Hirotani, K. Shibata, S., 1999a, MNRAS 308, 54 (Paper I)

Hirotani, K. Shibata, S., 1999b, MNRAS 308, 67 (Paper II)

Hirotani, K. Shibata, S., 1999c, PASJ 51, 683 (Paper III)

Hirotani, K. Shibata, S., 2001, MNRAS 325, 1228 (Paper VII)

Hirotani, K. Shibata, S., 2002a, ApJ 558, 216 (Paper VIII)

Hirotani, K. Shibata, S., 2002b, ApJ 564, 369 (Paper IX)

Hirotani, K., Harding, A. K., Shibata, S., 2003, accepted to ApJ (Paper X, astro-ph/0212043)

Kanbach, G., Arzoumanian, Z., Bertsch, D. L., Brazier, K. T. S., Chiang, J., Fichtel, C. E., Fierro, J. M., Hartman, R. C., et al. 1994, A \& A 289, 855

Kaspi, V. M., Lackey, J. R., Manchester, R. N., Bailes, M., Pace, R. 2000, ApJ 528, 445

Koribalski, B., Johnston, S., Weisberg, J. M., Wilson W. 1995, ApJ 441, 756

Mayer-Hasselwander, H. A., Bertsch, D. L., Brazier, T. S., Chiang, J., Fichtel, C. E., Fierro, J. M., Hartman, R. C., Hunter, S. D. 1994, ApJ 421, 276

Mestel, L. 1971, Nature Phys. Sci., 233, 149

Michel, F. C., 1974, ApJ, 192, 713

Mineo, T., Massaro, E., Cusumano, G., Becker, W. 2002, A\&A, 392, 181

Nolan, P. L., Arzoumanian, Z., Bertsch, D. L., Chiang, J., Fichtel, C. E., Fierro, J. M., Hartman, R. C., Hunter, S. D., et al. 1993, ApJ 409, 697

Ögelman, H., Finley, J. P., Zimmermann, H. U. 1993, Nature 361, 136

Pavlov, G. G., Zavlin, V. E., Sanwal, D., Burwitz, V., Garmire, G. P. 2001, ApJ, 552, L129

Ramanamurthy, P. V., Bertsch, D. L., Dingus, L., Esposito, J. A., Fierro, J. M., Fichtel, C. E., Hunter, S. D., Kanbach, G., et al. 1995, ApJ 447, L109

Romani, R. W. 1996, ApJ, 470, 469

Romani, R. W., Yadigaroglu, I. A. 1995, ApJ 438, 314

Scharlemann, E. T., Arons, J., Fawley, W. T., 1978 ApJ, 222, 297

Shibata, S. 1997, MNRAS 287, 262 
Shibata, S., Miyazaki, J., Takahara, F. 1998, MNRAS 295, L53

Shibata, S., Miyazaki, J., Takahara, F. 2002, MNRAS 336,233

Sturner, S. J., Dermer, C. D., Michel, F. C. 1995, ApJ 445,736

Takata, J., Shibata, S., Hirotani, K., 2002, in Proc. of University of Tokyo Workshop 2002 on The Universe viewed in Gamma-Rays, eds. Enomoto, R., Mori, M., Yanagita, S., in press.

Taylor, J. H., Cordes, J. M. 1993, ApJ 411, 674

Thompson, D. J., Bailes, M., Bertsch, D. L., Esposito, J. A., Fichtel, C. E., Harding, A. K., Hartman, R. C., Hunter, S. D. 1996, ApJ 465, 385

Thompson, D. J., Bailes, M., Bertsch, D. L., Cordes, J., D'Amico, N., Esposito, J. A., Finley, J., Hartman, R. C., et al. 1999, ApJ 516, 297

Yabe, T., Aoki, T. 1991 Comput. Phys. Commun., 66,219

Yabe, T, Xiao, F., Utsumi, T. 2001, J. Comput. Phys. 169,556

Zhang, L. Cheng, K. S. 1997, ApJ 487, 370

This 2-column preprint was prepared with the AAS LATEX macros v4.0.

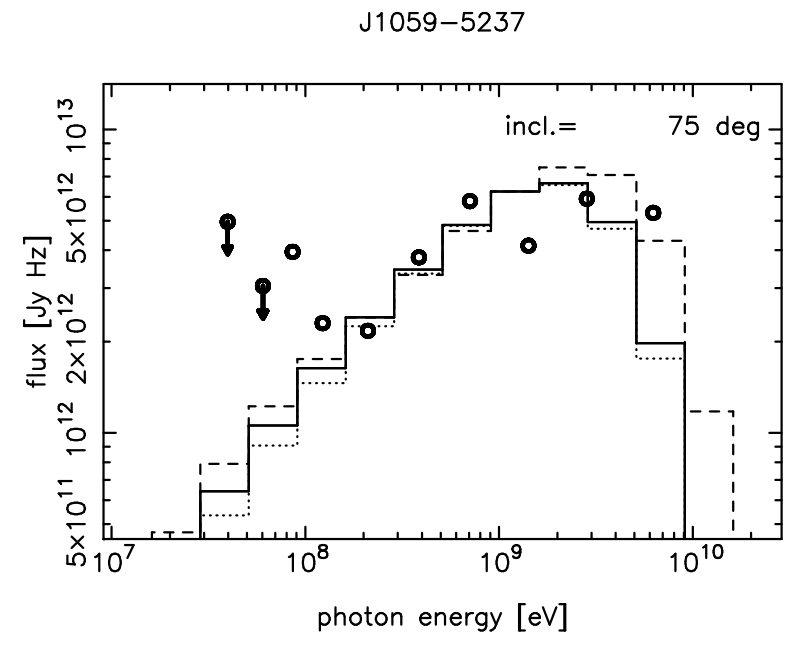

Fig. 10. - Computed $\gamma$-ray spectrum for B105552. Escaping particles little contribute for the luminosity. For the solid and dashed lines, $d=$ $1.53 \mathrm{kpc}$ is adopted; the corresponding parameter sets are $\left(j^{\text {in }}, j_{\text {gap }}, D_{\perp} / \varpi_{\mathrm{LC}}\right)=\left(0.25,2.9 \times 10^{-3}, 0.27\right)$, and $\left(0.125,3.7 \times 10^{-3}, 0.20\right)$, respectively. For the dotted line, $d=1 \mathrm{kpc}$ and $\left(j^{\text {in }}, j_{\text {gap }}, D_{\perp} / \varpi_{\mathrm{LC}}\right)=$ $\left(0.25,4.5 \times 10^{-3}, 0.16\right)$. 


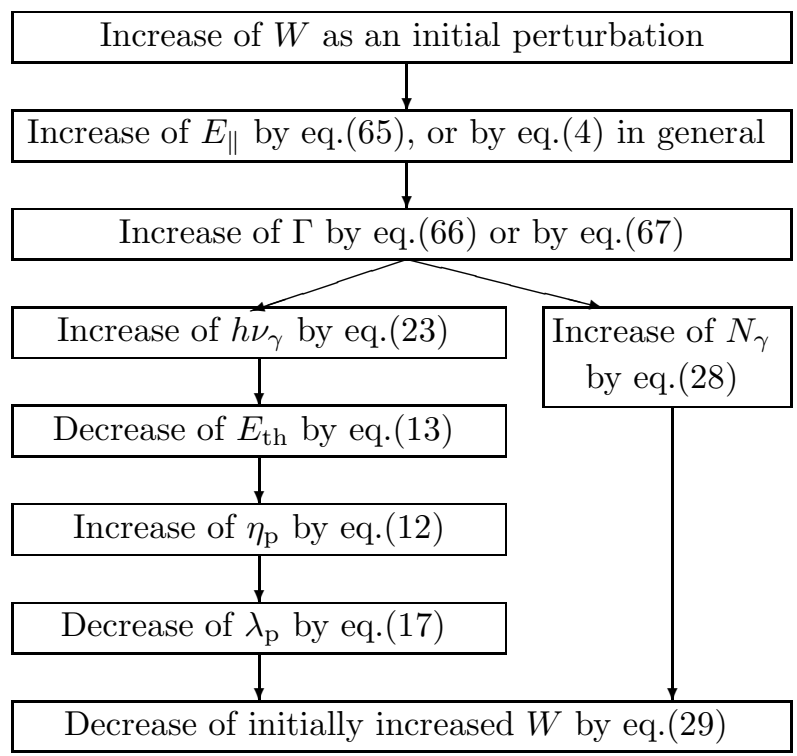

Fig. 11. - Stability of an outer-gap accelerator 